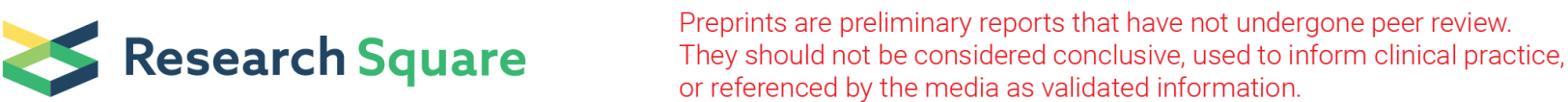

\section{A Short Shaker Channel Mediates K+ Loading to Root Conducting Tissues and Contributes to Rice Grain Yield under Field Conditions}

\section{Shunying Yang}

Institute of soil science, chinese academy of sciences

\section{Thanh-Hao Nguyen}

BPMP, Univ Montpellier, CNRS, INRAE, Institut Agro, Montpellier, France

\section{Cécile Fizames}

BPMP, Univ Montpellier, CNRS, INRAE, Institut Agro, Montpellier, France

\section{Junlin Li}

Shandong Institute of Sericulture, Yantai, China

\section{Sheliang Wang}

Microelement Research Centre, Huazhong Agricultural University

\section{Aurore Vernet}

CIRAD, UMR AGAP Institut, F-34398 Montpellier, France

\section{Emmanuel Guiderdoni}

Cirad

\section{Shaofei Wang}

State Key Laboratory of Soil and Sustainable Agriculture, Institute of Soil Science, Chinese Academy of Sciences

\section{Yanan Huang}

State Key Laboratory of Soil and Sustainable Agriculture, Institute of Soil Science, Chinese Academy of Sciences

\section{Dongli Hao}

State Key Laboratory of Soil and Sustainable Agriculture, Institute of Soil Science, Chinese Academy of Sciences

\section{Jiajin Wang}

State Key Laboratory of Soil and Sustainable Agriculture, Institute of Soil Science, Chinese Academy of Sciences

\section{Hervé Sentenac}

BPMP, Univ Montpellier, CNRS, INRAE, Institut Agro, Montpellier, France

\section{Anne-Aliénor Véry}

BPMP, Univ Montpellier, CNRS, INRAE, Institut Agro, Montpellier, France

Yan-Hua Su ( $\nabla$ yhsu@issas.ac.cn ) 
Institute of soil science, chinese academy of sciences

\section{Article}

Keywords: rice, short inward Shaker channel, K+ uptake capacity, root-shoot K+ transport, field grain yield

Posted Date: February 4th, 2022

DOI: https://doi.org/10.21203/rs.3.rs-1255684/v1

License: (c) (i) This work is licensed under a Creative Commons Attribution 4.0 International License.

Read Full License 


\section{Abstract}

$\mathrm{K}^{+}$uptake to root conducting cells is a prerequisite for its release to the xylem vessels and long-distance delivery to the aerial parts, though the molecular mechanism underpinning such process remains insufficiently understood. Here we report the discovery in rice root of an essential component involved in loading of $\mathrm{K}^{+}$to the stelar tissues encoded by an unusual short Shaker $\mathrm{K}^{+}$channel OsK2.2. OsK2.2 represents a novel type of short Shaker channels lacking the sequences immediately downstream of the putative cyclic nucleotide binding domain that present to the C-termini of all plant Shaker channels reported to date. In silico analysis suggests that such short Shaker channels are monocot-specific and especially found in the Poaceae species. Functional assessment in Xenopus oocytes reveals that OsK2.2 uses such innate $\mathrm{C}$-terminal shortness as an intrinsic strategy for maintaining large capacity of $\mathrm{K}^{+}$uptake to the cells. OsK2.2 predominantly localizes to the phloem and xylem peripheral cells of rice root vasculature. Disruption of OsK2.2 function causes approximately $30 \%$ lower $\mathrm{K}^{+}$in both phloem and xylem sap and reduces $\mathrm{K}^{+}$accumulation to the shoots. OsK2.2 strongly contributes to the grain yield of rice under field conditions. The essential contribution of such particular type of inward Shaker channel, operating in stelar tissues, to $\mathrm{K}^{+}$transport between roots and shoots and to grain yield, here evidenced in rice, may be a common trait amongst Poaceae species.

\section{Introduction}

In plants, $\mathrm{K}^{+}$is the most abundant and mobile cation. It is involved in electrical neutralization of the anionic groups of inorganic and organic anions and macromolecules, $\mathrm{pH}$ homeostasis, control of membrane electrical potential, and regulation of cell osmotic pressure. Through the latter function in plants, it plays a role in turgor-driven cell and organ movements, such as guard cell movements controlling stomatal aperture at the leaf surface and thereby $\mathrm{CO}_{2}$ fixation and plant transpirational water loss. Thus, plant development requires that large quantities of $\mathrm{K}^{+}$ions are taken up by roots from the soil and then transported upward and throughout the plant ${ }^{1,2}$.

Several families of transporters and channels have been identified in plants ${ }^{3,4}$. Genes of the Shaker family encode $\mathrm{K}^{+}$channels that dominate the plasma membrane conductance in most cell types and play major roles in massive $\mathrm{K}^{+}$transport across this membrane ${ }^{4}$. In Arabidopsis, for instance, KAT1 and KAT2 mediate the influx of $\mathrm{K}^{+}$into guard cells allowed by membrane hyperpolarization and lead to stomatal opening, while GORK mediates the efflux of $\mathrm{K}^{+}$resulting from membrane depolarization and leads to stomatal closure ${ }^{5-8}$. The AKT2 channels contribute to the distribution and recirculation of $\mathrm{K}^{+}$via the phloem vasculature ${ }^{9-11}$. In roots, the AKT1 Shaker channel contributes to $\mathrm{K}^{+}$uptake from the soil solution ${ }^{12,13}$; whereas SKOR mediates $\mathrm{K}^{+}$secretion into the xylem sap and translocation to shoots ${ }^{14}$.

The Shaker family is strongly conserved in plants, always displaying the same 5 sub-families in each species for a total number of members close to 10 in each genome (9 in Arabidopsis and 10 in rice) $)^{4}$. Homologs of the Arabidopsis KAT1/KAT2, GORK, AKT2, AKT1 and SKOR have been identified in 
rice $^{4,11,15-17}$ and in many other plant species, dicots and monocots, including for instance potato ${ }^{18}$, grapevine $^{19,20}$, maize ${ }^{21,22}$, barley ${ }^{23}$, poplar ${ }^{24}$ and Ammopiptanthus mongolicus ${ }^{25,26}$.

Shaker channels are tetrameric proteins, the four subunits, named alpha-subunits, can be the product of a single Shaker gene (homomeric channels) or of different Shaker genes (heteromeric channels) ${ }^{27,28}$. The assembly of the 4 alpha-subunits gives rise to a permeation pathway for $\mathrm{K}^{+}$in the center of the structure. An alpha-subunit typically displays a short cytosolic N-terminal domain, followed by the channel membrane hydrophobic domain and a long $\mathrm{C}$-terminal cytosolic region. The membrane hydrophobic domain comprises 6 transmembrane segments, named S1 to S6. The first four segments S1-S4 form a voltage-sensing module rendering Shaker channels regulated by voltage. The S5-S6 module forms the channel permeation pathway. The so-called pore loop (P), located between S5 and S6, harbors the canonical $\mathrm{K}^{+}$selectivity hallmark motif "TXGYGD". In the functional tetrameric protein, the $4 \mathrm{P}$ domains structure the aqueous pore at the center of the protein and render the permeation pathway highly selective for $\mathrm{K}^{+}$ion $^{29}$.

The cytosolic region downstream the membrane hydrophobic core is rather long and displays at least 2 domains in all the Shaker channels that have been characterized so far: a putative cyclic nucleotide binding domain (CNBD) and a domain named $\mathrm{K}_{\mathrm{HA}}$ because it is rich in hydrophobic and acidic residues ${ }^{30}$. A third domain displaying ankyrin repeats and named Anky can be identified between the CNBD and $\mathrm{K}_{\mathrm{HA}}$ domains in a majority of Shaker channels. Evidence has been obtained in the Arabidopsis AKT1 channel that the physical interactions between the $4 \mathrm{cNBD}$ domains within the tetrameric protein play a role in the tetramerization process ${ }^{31}$. The $\mathrm{K}_{\mathrm{HA}}$ domain would interact with the region lying between the channel hydrophobic core and the $\mathrm{CNBD}^{31}$. The latter interaction might contribute to the tetramerization process and/or to channel clustering in the membrane ${ }^{30,31}$. The AKT1 Anky domain has been shown to interact with regulatory protein partners, at least one member of the calcineurin B-like (CBL)-interacting protein kinases (CIPK), CIPK23. One CBL, CBL1 or CBL9, is involved in this interaction and regulates CIPK23 ${ }^{32-36}$.

In term of the long-distance transport toward the aerial parts, $\mathrm{K}^{+}$absorbed by epidermal cells must be moved laterally across the root cortex, loaded to the central stelar cells before being released to the xylem vessels. The SKOR channel, with its predominant localization to the Arabidopsis root stelle, represents a clearly defined component of the root-shoot $\mathrm{K}^{+}$transport pathway operating in secretion of $\mathrm{K}^{+}$to xylem vessels ${ }^{14}$. Knockout of the SKOR function reduces $\mathrm{K}^{+}$concentration in the xylem sap and causes halfway reduction of $\mathrm{K}^{+}$content in shoots, suggesting a major composition of the root-shoot transport of the ion $^{14}$. In a transverse view of the mechanisms of $\mathrm{K}^{+}$movement inside the root, it is clear only within the two terminal regimes: the initial $\mathrm{K}^{+}$absorption at root surface by the contributions of various high-affinity $\mathrm{K}^{+}$transporters and the AKT1 channels $4,13,16,29,37-40$ and the $\mathrm{K}^{+}$secreation to the xylem vessels enable by the SKOR channel ${ }^{14}$. Whereas the intermediate processes including $\mathrm{K}^{+}$translocation across cortical layers and loading to the conducting cells, remain elusive. In term of the central role of SKOR-mediated $\mathrm{K}^{+}$ 
secretion to xylem vessels of root, a question arises on the opposite direction, how $\mathrm{K}^{+}$is loaded to the conducting cells?

Here we report the identification of a new Shaker channel type whose sequence terminates just at the end of the cNBD domain and which is likely to be specific of monocots. The channel of this type that we have identified in rice, OsK2.2, is an inward rectifier. It is primarily expressed in the root stele where it contributes to $\mathrm{K}^{+}$translocation to shoots via the xylem sap, a function to which only outward rectifiers were reported to be associated until now. In addition, under field conditions, we observe that the function of OsK2.2 strongly contributes to grain yield of rice.

\section{Results}

\section{OsK2.2 encodes a Shaker $\mathrm{K}^{+}$channel harboring an unusually short C-terminus}

OsK2.2 (LOC_Os01g55200) was identified in the rice genome database (Rice Genome Annotation Project, http://rice.plantbiology.msu.edu) as a Shaker channel gene encoding a short polypeptide without any of the regions identified in typical plant Shaker channels downstream of the cNBD (Fig. 1a). The name OsK2.2 assigned to this sequence results from analyses of the structure of the plant Shaker family, which identified five subfamilies, this Shaker belonging to the subfamily \#2 $\left({ }^{15}\right.$, Fig. 1$)$. The deduced OsK2.2 polypeptide (502 amino acids) displays the classical hydrophobic core of Shaker $\mathrm{K}^{+}$channel built of six conserved transmembrane segments (named S1 to S6) (Fig. 1a), the hallmark pore-forming motif TxGYGD between S5 and S6 and the CNBD downstream the hydrophobic core ${ }^{29,39}$ (see Introduction). The protein terminates at the immediate end of the CNBD and is thereby shorter by approximately $150-200$ amino acids than the corresponding region in other Shaker subfamily \#2 members ${ }^{15}$ like the Arabidopsis AtKAT1 (Fig. 1a). When the sequence of the OsK2.2 channel (N-ter, hydrophobic core and cNBD domain) was aligned with the corresponding domains of other Shaker channels, the highest level of overall identity with the members of the Arabidopsis Shaker family was of about $65 \%$ with KAT1, the emblematic member of the Shaker subfamily \#2. The percentage of identity was of about $52 \%$ with the AKT1 channels from Arabidopsis AtAKT1 ${ }^{12}$ and rice OsAKT $1^{16}$. The C-terminal sequences present in AtKAT1 and OsAKT1 but absent from OsK2.2, have been named $\mathrm{C} 1$ and $\mathrm{C} 2$, respectively, in the following text. When compared with $\mathrm{C} 1, \mathrm{C} 2$ contains a series of ankyrin repeats since OsAKT1, like AtAKT1, harbors an Anky domain in contrast to KAT1 (Fig. 1a). A typical $\mathrm{K}_{\mathrm{HA}}$ domain, long of ca. 70 amino acids, is present in both $\mathrm{C} 1$ and $\mathrm{C} 2$ in the distal $\mathrm{C}$-terminus region of these polypeptides.

The fact that the identified OsK2.2 coding sequence corresponds to a complete cDNA was first validated by high-fidelity PCR amplification (Supplementary Fig. 1). When using rice cDNA and genomic DNA as templates, fragments of $1509 \mathrm{bp}$ (lane 1) and $2665 \mathrm{bp}$ (lane 2) were respectively obtained with the primer pair flanking the 5'-ATG and 3'-stop codon. These two fragments were exactly identical in size and sequence to those annotated in the rice genome database. Then, taking advantage of the poly $(A)$ tail of mature mRNAs, a further PCR experiment using the primer pair $5^{\prime}-A T G / 3^{\prime}$-oligo(dT) $)_{30}$ and rice cDNA 
templates yielded a $\sim 1.5 \mathrm{~kb}$ fragment (lane 3 ) containing the exact open reading frame (verified by sequencing) coding for 502 amino acids. Thus, although displaying an unusually short C-terminal sequence, innately without any region downstream of the cNBD, OsK2.2 is a complete protein. To our knowledge, OsK2.2 represents the shortest Shaker $\mathrm{K}^{+}$channel characterized in plants so far.

In silico searches were then carried out in order to take stock of such short Shaker channels in other plant species. The Shaker subfamily \#2 proteins AtKAT1, AtAKT1, OsK2.2, BdK2.2 and ZmK2.2 ${ }^{4}$ were used to identify their homologues (with BLASTP and an expect threshold of 1e-30) ${ }^{41}$ in all Viridiplantae species (taxid:33090) whose genomes are sequenced and annotated (NCBI refseq_protein database; September 2021). This yielded 1920 sequences, among which 1482 were annotated as belonging to a dicot species (the corresponding total number of dicot species being of 97), 332 were annotated as belonging to a monocot species (total number of monocot species: 21 ), and 83 simply identified as "flowering plants". Then, Prosite ${ }^{42}$ and CD-Search ${ }^{43}$ were used to check the presence/absence within each of these 1920 sequences of a full length transmembrane hydrophobic core (cl37996; Ion_Trans domain), of a cNBD domain (PS50042 domain; cNMP_binding domain), of an Anky domain (PS50088 or PS50271) and of a $\mathrm{K}_{\mathrm{HA}}$ domain (PS51490). A total of 61 sequences were then retained as displaying a cNBD domain but neither an Anky nor a $\mathrm{K}_{\mathrm{HA}}$ domain. These 61 sequences corresponded to 36 dicot sequences, for a total of 21 different dicot species, 24 monocot sequences for a total of 14 monocot species, and 1 archaic angiosperm. Then, with further investigation of these 61 sequences, the encoding genes were identified, which revealed that the initial pool of 61 sequences corresponded to 47 different genes. For each of these genes, all the variants present in the NCBI database were identified and analyzed in order to check the presence/absence of an Anky and/or a $\mathrm{K}_{\mathrm{HA}}$ domain in the longest variants. This yielded a set of 24 genes for which the longest encoded variant does not display any Anky or $\mathrm{K}_{\mathrm{HA}}$ domain. This set comprises 7 sequences from dicots, belonging to 7 different dicot species, and 17 sequences of monocots, for a total of 13 monocot species. The phylogenetic relationships of this set of 24 sequences with the 9 Shakers from Arabidopsis were analyzed (Fig. 1b). The 5 subfamilies identified within the plant Shaker family ${ }^{15}$ can be clearly distinguished within the phylogenetic tree. Most genes, 16 out of 24, identified as encoding short Shakers, without Anky and $\mathrm{K}_{\mathrm{HA}}$ domain, belong to the Shaker subfamily \#2. Interestingly, all these genes belong to monocot species, and no short Shaker from a dicot species is identified in this subfamily (Fig. 1b). The 7 dicot sequences (from the initial pool of 1482 sequences) identified as encoding Shakers without Anky and $\mathrm{K}_{\mathrm{HA}}$ domain belong to Shaker subfamilies \#3, 4 or 5, and not to subfamily \#2 (Fig. 1b).

The above analysis supports the hypothesis that short Shakers belonging to subfamily \#2, like OsK2.2, are specifically present in monocots. However, our searches for such channels (in the NCBI data base) did not identify such short subfamily \#2 Shaker sequence in several monocots, including Ananas comosus, Elaeis guineensis (oil palm), Musa acuminata (banana) and Phoenix dactylifera (date palm), which suggests that OsK2.2-like channels are not present in every monocot.

From the 12 monocot species identified as possessing at least one subfamily \#2 short channel (Fig. 1b), 11 belong to the Poacea family and one (Zingiber officinale) to the Zingiberacea family. For each of the 
11 Poceae, all the proteins belonging to the Shaker subfamily \#2 (i.e., whether they display a short Cterminus like OsK2.2 or a classical C-terminal region with a $\mathrm{K}_{\mathrm{HA}}$ domain) were collected from the NCBI refseq_protein database. Shaker subfamily \#2 channels were also collected for another Poacea, Hordeum vulgare (barley), from the NCBI non-redundant protein sequence (nr) database and added to the set of sequences collected from the other 11 Poacae. The phylogenetic relationships between the sequences thereby obtained show that 3 sub-groups can be identified within the Shaker subfamily \#2 in Poaceae (Fig. 1c). All the Poaceaes short subfamily \#2 channels belong to a single group and this group comprises only channels of this type. This group has thus been named GK2.2, by reference to OsK2.2. The 2 other groups, named GK2.1 and GK2.3, comprise channels displaying a classical C-terminal region in which a $\mathrm{K}_{\mathrm{HA}}$ domain can be identified. The fact that all short subfamily \#2 channels belong to the same phylogenetic group (GK2.2) suggests that the corresponding genes did not derive from events (resulting in the absence of $\mathrm{K}_{\mathrm{HA}}$ domain) that would have independently occurred in different species but that they have evolved from a common ancestor gene.

In conclusion, short members of the Shaker subfamily \#2, without $\mathrm{K}_{\mathrm{HA}}$ domain like OsK2.2, appear to be specific to monocots. They are especially found in Poaceae, in which a single channel of this type is present per genome (Fig. 1b,c) and where they have probably derived from a single common ancestor gene.

OsK2.2 probably uses its structural shortness as an intrinsic strategy for maintaining large capacity of $K$ + uptake activity

OsK2.2 was functionally characterized in Xenopus oocytes using the two-electrode voltage-clamp technique. In addition, in order to decipher the significance of the absence of a "classical" C-terminus (i.e., of a KAT1 or AKT1-type C-terminus) in OsK2.2, we also characterized 2 chimeric constructs, associating OsK2.2 to the $\mathrm{C} 1$ or $\mathrm{C} 2 \mathrm{C}$-terminal region present in AtKAT1 and OsAKT1, respectively (see Fig. 1a). The corresponding chimeric channels were named OsK2.2+C1 and OsK2.2+C2. Voltage steps ranging from -160 to $+20 \mathrm{mV}$ were applied to oocytes with an increment of $+10 \mathrm{mV}$, in presence of $50 \mathrm{mM} \mathrm{K}^{+}$in the bath solution ( $\mathrm{pH}$ 7.4). In OsK2.2 expressing oocytes, macroscopic inward currents were elicited at membrane potentials more negative than a threshold of approximately $60 \mathrm{mV}$. The inward current increased when the membrane potential was clamped at more negative values, and reached values as large as $-8 \mu \mathrm{A}$ at $-160 \mathrm{mV}$ (left panels in Fig. 2a,b). Such recordings indicate that OsK2.2 is functional despite its short $\mathrm{C}$-terminus, and gives rise to a bona fide voltage-gated inwardly-rectifying $\mathrm{K}^{+}$ conductance, like AtKAT1 and OsAKT1 16,44.

The OsK2.2+C1 and OsK2.2+C2 chimeras were also functional in Xenopus oocytes and behaved as inward rectifiers. Compared to OsK2.2, the presence of $\mathrm{C} 1$ or $\mathrm{C} 2$ in these chimeric constructs resulted however in ca. 50-60\% reduction in $\mathrm{K}^{+}$inward currents (Fig. 2a,b). It is worth noting that the reciprocal deletion of the corresponding $C 1$ sequence in AtKAT1 (AtKAT1 $\Delta C 1$ construct) led to 90-120\% increase in the current amplitude, when compared to the currents mediated by the wild-type AtKAT1 (Fig. 2a,b, right 
panels). Such differences between the chimeric constructs and OsK2.2, and between AtKAT1 and AtKAT $1 \Delta \mathrm{C} 1$, were repeatedly observed in independent experiments, with a total of at least 50 oocytes for each tested channel/construct. Statistical analysis of these pair-wised comparisons (Fig. 2b) provides evidence that the $\mathrm{C}$-terminal $\mathrm{C} 1$ and $\mathrm{C} 2$ sequences are intrinsically endowed with the capacity of downtuning the macroscopic current amplitudes. This reduction in macroscopic current amplitude reflects differences at least in the voltage sensitivity of the channels and in their level of expression and targeting to the cell membrane as highlighted here below.

With respect to the voltage sensitivity, analysis of the different channels in parallel experiments revealed that the fusion of $\mathrm{C} 1$ or $\mathrm{C} 2$ to OsK2.2 resulted in a shift of the channel activation curve towards more negative values, the half-activation potential being shifted by 16 or $19 \mathrm{mV}$ (Fig. 2c, left panel). Conversely, the deletion of $\mathrm{C} 1$ from AtKAT1 shifted the half-activation potential towards more positive values by $\sim 28$ $\mathrm{mV}$ (Fig. 2c, right panel). Thus, the presence of $\mathrm{C} 1$ or $\mathrm{C} 2$ terminus results in smaller $\mathrm{K}^{+}$currents at given membrane potentials. The amounts of channels present at the cell membrane were assessed by fusing an enhanced GFP protein (eGFP) to the C-terminal end of OsK2.2, OsK2.2+C1 and OsK2.2+C2. The amounts of GFP fluorescence associated to the cell membrane were reduced by the presence of $\mathrm{C} 1$ or $\mathrm{C} 2$ in the chimeric constructs (Fig. 2d), suggesting that the absence of $\mathrm{C} 1$ and $\mathrm{C} 2$ type $\mathrm{C}$-terminal sequence facilitates OsK2.2 expression and targeting to the plasma membrane in oocytes. Altogether, these data support the hypothesis that the shortness of the Cterminal cytosolic sequence of OsK2.2 contributes to the intrinsic capacity of this channel to mediate large $\mathrm{K}^{+}$currents.

In a last set of electrophysiological analyses in Xenopus oocytes, we tested the sensitivity of OsK2.2, OsK2.2+C1, OsK2.2+C2 and of a third chimeric construct, OsK2.2+C1+Anky (OsK2.2+C1 in fusion with the Anky domains of OsAKT1) to the regulatory complex OsCBL1/OsCIPK23. In rice, this complex activates OsAKT1 ${ }^{16}$ in a similar way as AtCBL1/AtCIPK23 activates AtAKT1 in Arabidopsis ${ }^{32}$. Neither OsK2.2 nor OsK2.2+C1 was found to be regulated by the OsCBL1/OsCIPK23 complex, while the 2 Anky domaincontaining constructs OsK2.2+C2 and OsK2.2+C1+Anky were activated by this complex (Fig. 3). Besides providing direct evidence that OsK2.2 activity is not under control of the CBL/CIPK complex, these results provide further support to the hypothesis that regulation of channel activity by this type of complex requires the presence of an Anky domain within the channel sequence. They also indicate that the regulatory process does not involve a unique and fixed channel structure since the presence of an Anky domain downstream of the $\mathrm{K}_{\mathrm{HA}}$ domain, and not upstream as in Shaker channels harboring such a domain, is sufficient for the regulatory interactions to take place. A shift in the half-activation potential by about $30 \mathrm{mV}$ toward more positive voltages resulted from the activation of the construct OsK2.2+C1+Anky by the OsCBL1/OsCIPK23 complex (Fig. 3d).

\section{OsK2.2 is primarily expressed in root stellar tissues}

Real-time quantitative RT-PCR analyses revealed that OsK2.2 was predominantly expressed in rice roots and very weakly in shoots (Fig. 4a). In roots, reporter gene experiments using a 2264 bp promoter-driven GUS construct indicated that the expression of OsK2.2 was concentrated in stellar tissues of both 
seminal (Fig. 4b) and lateral (Fig. 4c) roots. In shoots, weak GUS reporter gene activity was also observed in guard cells (Fig. 4d). In situ PCR using specific primers and digoxigenin-11-incorporated dUTP allowed DIG-labeled amplification of OsK2.2 transcripts in $60 \mu \mathrm{m}$-thick transverse slices of rice roots. Subsequent immuno-detection with anti-DIG antibodies and BM purple staining revealed an exclusive localization within the stele, in phloem and xylem peripheral cells of the root vasculature (Fig. 4e). Such an expression pattern suggested a role in $\mathrm{K}^{+}$transport in root vascular tissues.

\section{OsK2.2 plays a role in long distance $\mathrm{K}^{+}$transport in root vascular tissues}

To investigate the physiological roles of OsK2.2, osk2.2 gene mutation lines (Nipponbare background, NB) were created using the CRISPR/Cas9 gene editing technique. Two independent mutant lines were obtained with nucleotide deletions in the first exon of the gene: osk2.2-1, displaying the deletion of 2 " $\mathrm{A}$ " at positions 148 and 149, and osk2.2-2 displaying a single A deletion at position 148 (Supplementary Fig. 2a). In both cases, the shift of the open reading frame caused by the deletion led to early termination of protein translation, at amino acid (aa) 58 for osk2.21 and 69 for osk2.22, the aa positions 58 and 69 being located upstream from the first predicted transmembrane segment $\mathrm{S} 1$. Thus, both mutations resulted in the loss of OsK2.2 functional expression (KO mutant plants). In parallel, rice cultivar Zhonghua-11 (ZH11) was transformed with an ubiquitin promoter-driven OsK2.2 construct to generate overexpression $(O E)$ lines. Two individual lines, $O E \# 3$ and $O E \# 17$, respectively displaying 35 - and 28 - fold expression levels in roots (33 and 30 fold respectively in shoots) were obtained (Supplementary Fig. 2b). Homozygous mutant and $O E$ lines were propagated, screened and used for investigating the physiological functions of OsK2.2.

Fourteen-day-old rice seedlings grown in $1 \mathrm{mM} \mathrm{K}^{+}$modified IRRI (International Rice Research Institute, the Philippines) solution were starved in $\mathrm{K}^{+}$-free nutrient solution for 3 days before being treated for $3 \mathrm{~h}$ in 20 $\mathrm{mM} \mathrm{K}^{+}$. No significant difference in biomass was observed between the wild-type control plants and the corresponding KO mutant and $O E$ seedling grown in these conditions (not shown). Phloem and xylem sap samples were then collected at the end of the $3 \mathrm{~h}$ treatment in $20 \mathrm{mM} \mathrm{K}^{+}$. The amounts of $\mathrm{K}^{+}$assayed in the phloem sap samples were lower by ca. $30 \%$ or $40 \%$ in the osk 2.21 and 0 sk $2.22 \mathrm{KO}$ mutant seedlings and larger by ca. $15 \%$ or $22 \%$ in the $O E \# 3$ and $O E \# 17$ seedlings, when compared with the respective wildtype control seedlings (Fig. 5a). Regarding the xylem transport, the concentration of $\mathrm{K}^{+}$in the xylem sap samples was lower by $26-32 \%$ in the osk 2.21 and osk 2.22 mutant plants, and higher by $12-14 \%$ in the OE plants, when compared with the respective WT control plants (Fig. 5b). These data indicated that the long distance transport of $\mathrm{K}^{+}$in both the xylem and phloem vasculature is dependent on OsK2.2 functional expression.

A plant growth phenotype was apparent in seedlings initially grown for 10 days in $1 \mathrm{mM} \mathrm{K}^{+}$and then transferred for 7 further days in $10 \mathrm{mM} \mathrm{K}^{+}$(Fig. 6a): the shoot biomass was significantly lower, by about $22 \%$ and $16 \%$, in the osk 2.21 and osk 2.22 mutant plants than in the corresponding WT control plants. 
Whereas the root $\mathrm{K}^{+}$contents were similar in the mutant and WT plants, the shoot $\mathrm{K}^{+}$contents were significantly lower in the mutant plants, by $15-20 \%$ (Fig. $6 \mathrm{~b}$ ). The total amounts of $\mathrm{K}^{+}$present in shoots (total accumulation of $\mathrm{K}^{+}$in shoots), taking into account the differences in shoot biomass, were lower by $34 \%$ and $32 \%$ in the osk2.21 and osk2.22 mutant plants, compared to the WT plants (Fig. 6d). Altogether, these data indicated that OsK2.2 activity resulted in increased $\mathrm{K}^{+}$net translocation to shoots under these experimental conditions. The results obtained with the two $O E$ lines, $O E \# 3$ and $O E \# 17$ in the same conditions (in parallel experiments) were consistent with the above conclusion: the total amounts of $\mathrm{K}^{+}$ present in shoots was higher in these plants, compared to the corresponding WT control plants (Fig. 6d), the difference being essentially due to significantly larger shoot $\mathrm{K}^{+}$contents (Fig. 6c) since the overexpression of OsK2.2 was found to poorly affect the shoot biomass in these conditions (Fig. 6a). Finally, the large differences in total accumulation of $\mathrm{K}^{+}$in shoots indicated that $O s K 2.2$ functional expression resulted in increased net $\mathrm{K}^{+}$uptake from the external solution (containing $1 \mathrm{mM} \mathrm{K}^{+}$) by the root system although the expression of this gene in roots is restricted to stellar tissues.

\section{Multiple contributions of OsK2.2 functional expression to the plant phenotype}

Instantaneous net $\mathrm{K}^{+}$influxes were measured in lateral roots, at about $2 \mathrm{~mm}$ from the apex, from 10-dayold rice seedlings using the so-called Non-invasive Micro-Test technique (NMT) in a bath solution containing $1 \mathrm{mM} \mathrm{K}^{+}$. Negative values of the measured $\mathrm{K}^{+}$fluxes indicate that net $\mathrm{K}^{+}$uptake was achieved by the roots under these conditions. The rates of local net $\mathrm{K}^{+}$uptake were decreased by about $50 \%$ in both osk2.2 mutant roots (Fig. 7a) and were about 2-times higher in the OE roots (Fig. 7b) when compared to the rates recorded in the corresponding WT plants. Considering the dominant vasculature localization described above (Fig. 4), these data suggest that efficient long-distance $\mathrm{K}^{+}$transport in the xylem and phloem vasculatures favors $\mathrm{K}^{+}$uptake at the root surface.

Efficient $\mathrm{K}^{+}$acquisition by roots, $\mathrm{K}^{+}$accumulation in shoots and control of the $\mathrm{K}^{+} / \mathrm{Na}^{+}$content ratio in shoots are major determinants of plant salt stress tolerance. In order to evaluate the contribution of OsK2.2 activity to salt stress tolerance in rice, 10-day-old rice seedlings were subjected for 14 days to saline conditions by adding $100 \mathrm{mM} \mathrm{NaCl}$ into the hydroponic solution (Fig. 8a,b). The shoot biomass was found to be reduced by $7-19 \%$ in the osk 2.21 and osk 2.22 mutant plants, when compared to the respective WT, whereas it was increased by about $25 \%$ in the OE lines (Fig. 8c). Significantly lower $\mathrm{K}^{+}$ contents in shoots (by 18\% 19\%) and total $\mathrm{K}^{+}$accumulation in shoots (by $-25 \% \sim 33 \%$ ) were displayed by the mutant plants while the $O E$ lines displayed higher $\mathrm{K}^{+}$contents $(+23 \sim 42 \%)$ and accumulation (+53 81\%) when compared with the respective WT controls (Fig. 8d,e). In contrast, regarding $\mathrm{Na}^{+}$, larger $\mathrm{Na}^{+}$contents $(+40 \sim 60 \%)$ and total accumulation (+28 40\%) were determined in the KO mutant shoots (Fig. 8f), while lower $\mathrm{Na}^{+}$contents $(-8 \% \sim 11 \%)$ and total accumulation $(-14 \% \sim 17 \%)$ were assayed in the OE shoots (Fig. 8g), when compared with the corresponding WT values. These differences in $\mathrm{K}^{+}$and $\mathrm{Na}^{+}$ contents mean that the $\mathrm{K}^{+} / \mathrm{Na}^{+}$shoot content ratio was strongly lower, by ca. $45 \%$, in the osk $2.2 \mathrm{KO}$ mutant plants when compared to the corresponding WT control plants, while it was higher by about $42 \%$ 
in the $O E$ plants (Fig. 8h). Thus, this whole set of results indicated that OsK2.2 activity contributed to salt stress tolerance and that this beneficial effect could be ascribed, at least in part, to improved shoot $\mathrm{K}^{+} / \mathrm{Na}^{+}$homeostasis. The increase in $\mathrm{Na}^{+}$translocation to shoots in the absence of OsK2.2 activity in the mutant plants might result from the fact that the resulting reduction in inward $\mathrm{K}^{+}$channel activity in some cell types, where OsK2.2 is expressed in WT plants, is likely to lead to more polarized membrane potentials favoring $\mathrm{Na}^{+}$uptake.

In a last series of experiments, photosynthetic parameters were measured in the 2nd last leaves of 14day-old rice seedlings deprived of $\mathrm{K}^{+}$for 3 days and thereafter resupplied with $20 \mathrm{mM} \mathrm{K}^{+}$(same experimental conditions as those used for the experiment described by Fig. 5). The stomatal conductance (Gs), photosynthesis $(\mathrm{Pn})$ and transpiration (Tr) rates were reduced by $16 \sim 18 \%, 22 \sim 26 \%$ and $28 \sim 32 \%$, respectively, in osk2.2 mutant seedlings while these physiological parameters were increased by ca. $15 \%-25 \%, 30 \%-35 \%$ and $15 \%-30 \%$ in the OE seedlings when compared with the respective WT seedlings (Fig. 9). Thus, OsK2.2 also contributed to control of plant transpiration and photosynthesis, which probably involved, at least in part, its activity in guard cells (Fig. 4d).

\section{OsK2.2 functions strongly contribute to rice grain yield under field conditions}

The contribution of OsK2.2 to rice grain production in field conditions was assessed in 2 independent growth seasons, in 2017 and 2019 (Fig. 10). The osk2.2 mutant plants, compared to the corresponding WT plants, displayed dramatic growth and yield impairments, as revealed by a reduction in shoot biomass by about $40 \%$, a decrease in the number of seeding tillers per plant by about $25 \%-30 \%$ and finally a grain yield loss by about $40 \%$ (Fig. 10a,b). In contrast, approximately $20 \%$ increases in shoot biomass, seeding tillers and grain yield were observed in the $O E$ plants (Fig. 10a,c). At the late stage of grain filling, the flag leaf $\mathrm{K}^{+}$contents were $20-25 \%$ lower and $15 \%$ higher in the mutant and $O E$ lines, respectively, than in the corresponding WT plants (Fig. 10d). At this stage, the flag leaf nitrogen contents were lower by about $18 \%$ in the osk 2.2 mutant plants and higher by about $17 \%$ in the $O E$ plants (Fig. 10e).

\section{Discussion}

\section{The monocot Shaker channel subfamily \#2 comprises short members like the rice OsK.2.2}

Five subfamilies of Shaker channels have been identified in plants based on phylogenetic analyses and functional properties. With respect to functional properties, the members from subfamilies \#1 and \#2 are inwardly-rectifying $\mathrm{K}^{+}$channels. They differ by the presence or the absence (subfamily \#1 and \#2, respectively) of an Anky domain downstream of the cNBD domain. Classical representatives of these 2 subfamilies in Arabidopsis are AKT1 (subfamily \#1) and KAT1 (subfamily \#2). Subfamily \#3 (a single member in Arabidopsis: AKT2) corresponds to weakly rectifying channels and subfamily \#5 members 
(SKOR and GORK in Arabidopsis) are outwardly rectifying channels. Subfamily \#4 (a single member in Arabidopsis: KC1) includes regulatory subunits that do not form functional channels by themselves (at least in Arabidopsis) but are able to interact with members from subfamilies \#1, 2 and 3 to give rise to heterotetrameric channels with modified functional properties. At the structural level, the main difference between the members of these 5 subfamilies is the presence (in subfamilies \#1, 3 and 5) or the absence (subfamilies \#2 and 4) of an Anky domain.

Functional and phylogenetics analyses both indicate that OsK2.2, qualified of "short" Shaker channel when compared with the other Shaker channels characterized so far since its sequence ends immediately after the cNBD (Fig. 1a), is abona fide inwardly rectifying channel (Fig. 2a) and that it is phylogenetically related to subfamily \#2 members (Fig. 1b). Thus, OsK2.2 belongs to Shaker subfamily \#2. Phylogenetic analyses indicate that other short Shaker channels that can be identified in silico, like HvK2.2, BdK2.2, ZmK2.2 or TdK2.2, belonging to the subfamily \#2. Thus, this subfamily comprises KAT1-type "long" channels, displaying a rather long sequence downstream the cNBD, of about 150 aa and including a $\mathrm{K}_{\mathrm{HA}}$ domain, together with OsK2.2-type short channels, ending just after the CNBD, the two types of channels being inward rectifiers. Hence, the five-group distribution of the Shaker $\mathrm{K}^{+}$channel family in higher plants ${ }^{4,15}$ remains compatible with the identification of a significantly shortened structural type that ends just after the cNBD.

Our in silico analyses suggest that Shaker channel genes belonging to subfamily \#2 and displaying a sequence that encodes a channel ending just downstream of the cNBD are specific to monocots. For instance, sequences of this type can be found in barley, maize, wheat or Brachypodium distachyon and Sorghum bicolor (Fig. 1b,c), but undoubtedly not in Arabidopsis, poplar and Medicago truncatula. Of course, it cannot be excluded that dicots express such channels via, for instance, alternative splicing events. It is however worth noting that the searches we did for mRNA sequences encoding "short" Shaker subfamily \#2 channels ending just downstream of the cNBD have not allowed to identify any mRNAs of this type in dicot species. It is also worth noting that all the Shaker genes that can be identified in the moss Physcomitrella patens and in the fern Selaginella moellendorffii (4 and 3 genes, respectively) display an Anky domain in their C-terminal region downstream of a cNBD, suggesting that the OsK2.2 gene ancestor derived from a gene encoding a channel harboring a long $\mathrm{C}$-terminus and that this evolution might have occurred in monocots only.

The Shaker subfamily \#2 often comprises more than 1 member per genome, e.g., 2 in Arabidopsis, 3 in rice, barley and Brachypodium distachyon, and 4 in maize (Fig. 1C). In all of these monocots, the subfamily \#2 systematically comprises one and only one member corresponding to the OsK2.2 short type. This strongly suggests that, in each of these species, other members from subfamily 2 cannot substitute for the short member. It is thus likely that the subfamily \#2 short member plays a series of roles requiring special functional properties and/or regulation processes that the other Shaker channels do not display. 
OsK2.2 probably uses the C-terminal shortness as a strategy for maintaining large capacity of $\mathrm{K}^{+}$uptake activity

The function of $\mathrm{C}$-termini of Shaker $\mathrm{K}^{+}$channels has been proposed by deletion and mutagenesis studies for the contribution to the channels' voltage sensitivity ${ }^{10}$, tetramerization ${ }^{31}$ and / or heteromerization between channels ${ }^{45}$. However, OsK2.2 is an inherited shortest Shaker encoded only by 502 amino acids but is fully functional (Fig. 2). According to our results obtained in Xenopus oocytes, the shortness of the C-terminal sequences allows OsK2.2 more capable of $\mathrm{K}^{+}$uptake as compared to its mutations of Cterminal completeness (Fig. 2). This phenomenon can be apparently reproduced with the Arabidopsis AtKAT1 by parallel C-terminal deletion (Fig. 2), allowing us to propose a role of the distal C-termini in tuning the channels' activity and shaping the voltage sensitivity among KAT-type channels. As compared to the 'long' channels, i.e. OsK2.2 completed with the 'missing' C-terminal sequences and the wildtype AtKAT1, the short channels without the presence of the corresponding C-termini (OsK2.2 wildtype and AtKAT $1 \Delta \mathrm{C}$ ) are more capable of mediating $\mathrm{K}^{+}$uptake currents and particularly, the shift of channels' activation toward the positive voltages provides the possibility that the short channels like OsK2.2 is able to operate to allow significant $\mathrm{K}^{+}$influx into cells at a membrane voltage range around the cells' resting membrane potentials (Fig. 2). This mode-of-action can be further interpreted by enhanced intensity of the OsK2.2 channel proteins targeted on the oocyte membranes as traced by the eGFP-fusion constructs (Fig. 2e). In addition, considering the possibility that the short channel OsK2.2 may be evolved from an Ankycontaining ancestors such as AKT1 of the subfamily \#2, which uses a universal CBL/CIPK modulation pathway for tuning its $\mathrm{K}^{+}$uptake activity ${ }^{16,32-36}$, the shortness of the distal C-terminus with OsK2.2 may represent a structural strategy that skips such regulation, while strong $\mathrm{K}^{+}$uptake capability is maintained (Fig. 3). Altogether allows us to suggest that OsK2.2 probably uses its $\mathrm{C}$-terminal shortness as a unique structural strategy for maintaining persistently strong capability of mediating $\mathrm{K}^{+}$uptake and / or even at the membrane voltage range around the cells' resting potentials. Prospectively, while obviously needs to be further investigated, the action strategy described here for OsK2.2 may be a common trait for the short type of Shaker channels identified in silico amongst especially Poaceae species.

\section{OsK2.2 is a newly discovered component of the long distance $\mathrm{K}^{+}$transport}

$\mathrm{K}^{+}$loading to root xylem is a critical step for its long distance transport toward the aerial parts ${ }^{46,47}$. Since a long time peroid the root stele outward rectifier SKOR has been considered as the only Shaker channel with a specific role in root-shoot $\mathrm{K}^{+}$transport by secreting $\mathrm{K}^{+}$from the root stele conducting cells to the xylem vessels ${ }^{14}$. More recently, other Shaker channels and high-affinity $\mathrm{K}^{+}$transporters of the KT/HAK/KUP family have been proposed for a contribution, while not specifically according to their localizations, to the root-shoot $\mathrm{K}^{+}$transport particularly under low $\mathrm{K}^{+}$supply or salt stress conditions. Examples are the OsK5.2 channel of rice ${ }^{17}$, AtKUP7 of Arabidopsis ${ }^{48}, \mathrm{OsHAK}^{49}{ }^{49}, \mathrm{OsHAK}^{50}$ and OsHAK $21^{51}$ of rice. Because of the close link among the uptake capacity at the root surface, the crosstissue translocation within the root and the long-distance delivery through the xylem pathway, the 
impairment in $\mathrm{K}^{+}$uptake especially under $\mathrm{K}^{+}$limitation would to certain extent be expected to result in an effect on the efficiency of the root-shoot transport of this ion. Also considering the high millimolar concentration ranges of apoplastic $\mathrm{K}^{+}$inside the plant, the high-affinity transporters would be expected to contribute but not dominantly to the transport of $\mathrm{K}^{+}$through the long distance root-shoot pathway. With this respect, particularly in specific coordination with a SKOR-mediated $\mathrm{K}^{+}$secretion process from the root stele conducting cells, a mechanism acts on the opposite direction-to load these cells, has not been identified to date. A much unexpected role of the OsK2.2 $\mathrm{K}^{+}$inward rectifier, that was not reported for any inward rectifier so far, is the contribution to $\mathrm{K}^{+}$long distance transport from roots to shoots via the xylem sap (Fig. 5b). First, in root, the localization of OsK2.2 to the phloem and xylem parenchyma cells to a great extent overlaps that of SKOR (Fig. 4). Second, OsK2.2 acts as a inwardly rectifying $\mathrm{K}^{+}$uptake channel (Fig. 2) and is acutely involved in the $\mathrm{K}^{+}$transport toward the shoots (Fig. 5). Taking in account for its stele localization, OsK2.2 is thus proposed for a role in loading the stele cells, a precisely functional match to the SKOR pathway. Third, in the same parenchyma cells for instance, the opposite direction of the action of both channels in term of changes in membrane potentials could form a bilateral facilitation to sustained the function of each other: the operation of SKOR-mediated $\mathrm{K}^{+}$efflux from root stele hyperpolarizes the conducting cells and prevents the sustained action of the SKOR channel but favors the activation of OsK2.2 channel's $\mathrm{K}^{+}$uptake into these cells; as a consequence of $\mathrm{K}^{+}$influx through OsK2.2, the membrane potential of conducting cells restores back to the depolarization status required for the operation of SKOR. Thus continous $\mathrm{K}^{+}$loading / release of the conducting cells is sustained. Therefore, OsK2.2 composes of a new and specific component of the root-shoot $\mathrm{K}^{+}$transport system that operates in close coordination with SKOR.

\section{The function of OsK2.2 contains a strong relevance to the grain yield of rice}

The present data indicate that OsK2.2 functional expression in rice plays a direct or indirect role in diverse functions related to $\mathrm{K}^{+}$transport in the plant, root $\mathrm{K}^{+}$uptake, $\mathrm{K}^{+} / \mathrm{Na}^{+}$homeostasis under saline conditions and tolerance to salt stress, and transpiration and photosynthesis via probably at least a role in control of stomatal conductance. Taken in account that the importance of $\mathrm{K}^{+}$as a major mineral nutrient to overall plant health and development, the genetic modification of $\mathrm{K}^{+}$transport systems by creating transgenic or targeted gene-editing crops is expected to be a valuable approach to the molecular breeding for the improvement of crops' yield. Whereas the attempts on engineering $\mathrm{K}^{+}$channels have mostly been limited to the laboratory experiments for the purposes of deciphering their physiological relevances. Therefore, we realize such assessment under the field growth conditions that challenge the naturally environmental fluctuations. In two independent experiments, carried out in 2017 and 2019, a considerable contribution of OsK2.2 to grain yield was observed (Fig. 10a,b). Diverse consequences of the absence of OsK2.2 functional expression were also revealed by these field experiments, such as a reduction in the number of seeding tillers and of flag leaf $\mathrm{K}^{+}$and $\mathrm{N}$ contents (Fig. 10), which enlarges the spectrum of the indirect consequences of this mutation. Together with the results of the field experiments, as well as the data 
showing that OsK2.2 plays a role in salt stress tolerance, the fact that cereals of major importance, such as maize, wheat and barley, always possess one (and only one per genome) short member within their Shaker subfamily \#2 should increase the interest for this type of channel. Prospectively, besides the direct generation of transgenic crops by overexpressing the $\mathrm{K}^{+}$channel genes, the short channel of OsK2.2 evidenced in rice provides a potentially useful approach for the targeted gene editing toward the improvement of crops' $\mathrm{K}^{+}$nutrient use efficiency and productivity.

In summary, the predominant activity of loading the stelar cells allows us to suggest OsK2.2 as a newlydiscovered component of long distance $\mathrm{K}^{+}$transport in rice roots that is closely compatible and coordinates with the operation of the SKOR pathway. And the structual strategy of OsK2.2 for maintaining highly capable of $\mathrm{K}^{+}$uptake activity and its strong contribution to the grain yield of rice under field conditions provide a potentially useful target for genetic modifications of crops through gene editing.

\section{Methods}

\section{Molecular cloning and rice mutant production}

The coding sequences (CDS) of OsK2.2 (LOC_Os01g55200), OsAKT1 (LOC_Os0g45990), OsCBL 1 (LOC_Os10g41510), OsCIPK23 (LOC_Os07g05620) and AtKAT1 (At5g46240) were obtained by highfidelity PCR amplification from rice (cultivar Nipponbare) and Arabidopsis (Columbia ecotype) cDNA libraries, respectively, and verified by sequencing. The construct encoding the $\mathrm{C}$-terminus truncated AtKAT1 (AtKAT1 $\triangle C 1$, equivalent to OsK2.2 with respect to the length and structure of the C-terminal region downstream of the putative CNBD; Fig. 1a) was obtained by high-fidelity PCR amplification against the full-length AtKAT1 clone using primers allowing to omit the C-terminal sequence corresponding to amino acids 501-677. The sequences encoding the C-termini from AtKAT1 (C1, amino acids 501-677) and OsAKT1 (C2, amino acids 541-935) were obtained by PCR and fused to OsK2.2 to "complete" this channel with a longer $\mathrm{C}$-terminal region like in "classical" types of Shaker channels. The resulting chimeric channels were named OsK2.2+C1 and OsK2.2+C2. The Anky domain of OsAKT1 (Anky, amino acids 565757) was similarly amplified and fused to the OsK2.2+C1 construct. The resulting channel (displaying an

Anky domain downstream of the $\mathrm{K}_{\mathrm{HA}}$ domain) was named OsK2.2+C1+Anky. In addition, an enhanced GFP coding sequence (eGFP) was fused to the C-terminus of OsK2.2 and of its related chimeric constructs OsK2.2+C1 and OsK2.2+C2, to assess the abundance of the encoded proteins at the oocyte membrane. The CDS of the above channels and recombined chimeras were cloned into the oocyte expressing vector $\mathrm{pCl}$. OSCBL 1 and OSCIPK23 coding sequences were cloned and introduced into the pT7TS vector for in vitro cRNA synthesis.

OsK2.2 gene promoter region (2264 bp), amplified by PCR from rice cv Nipponbare genomic DNA and checked by sequencing, was introduced into the plant expression vector pCambia1301 upstream of the GUS reporter gene, replacing the CAMV35S promoter. Transgenic plants expressing the GUS reporter gene 
under control of the OsK2.2 promoter region were obtained through Agrobacterium-based transformation, as previously described ${ }^{17}$.

Targeted gene editing by the CRISPR-Cas 9 technique was used for the generation of osk2.2 knockout mutant rice lines. A gene-specific spacer hybridizing to the CDS of OsK2.2 was selected from rice genespecific spacer library ${ }^{52}$. The sgRNA expression cassette was prepared in vitro by PCR amplification of the ligated cassette, and then the sgRNA expression cassette was introduced into the final expression vector pBWA (VH-Cas9ir)-OsK2.2 using the Golden Gate technology 53,54 . For the overexpression construct, the CDS of OsK2.2 was inserted into the pUN1301 vector (vector pCambia1301 modified with ubiquitin

promoter instead of cauliflower mosaic virus $35 \mathrm{~S}$ promoter, according to Wang's description ${ }^{55}$. Subsequent plant transformations were carried out by a commercial company (Wuhan Biorun Bio-Tech Co., Ltd, China). Briefly, the plasmids were first introduced into Agrobacterium tumefaciens EHA105 strain and then transformed into calli derived from mature seeds of Oryza sativa ssp. Japonica cultivar Nipponbare (NB) for the knockout mutant lines, or from rice cultivar Zhonghua-11 (ZH-11) for the OE lines, and seedlings were regenerated by tissue culture. Primers used in these experiments are listed in Supplementary Table 2.

\section{Gene expression localization analysis}

For histochemical analysis of GUS activity in the root, 1-week-old T2 homozygous plants, screened by hygromycin resistance of their offspring, were grown in Yoshida hydroponic medium ${ }^{56}$. All samples were immerged in GUS staining solution (50 mM phosphate buffer, $\mathrm{pH}$ 7, $2.5 \mathrm{mM}$ ferricyanide, $2.5 \mathrm{mM}$ ferrocyanide, $0.05 \%$ [v/v] Triton X-100, and $1 \mathrm{mM} \mathrm{X-Gluc)} \mathrm{contained} \mathrm{in} \mathrm{a} \mathrm{24-well} \mathrm{plate,} \mathrm{the} \mathrm{leaves} \mathrm{having}$ been previously quickly divided into approximately $1 \times 1-\mathrm{cm}$ pieces. The plate was placed under vacuum for 30 min to facilitate the penetration of the GUS staining solution into the tissues, and the samples were incubated overnight at $37^{\circ} \mathrm{C}$. Leaf samples were then washed with $70 \%$ ethanol solution to remove chlorophyll and overnight treated with chloral hydrate $(2.5 \mathrm{mg} / \mathrm{L}$ in $30 \%$ glycerol) before being observed under microscope (BH2; Olympus) for GUS activity detection. A RM 2165 microtome (Leica) was used to obtain thin sections ( $8 \mu \mathrm{m}$ thick) from stained fixed samples (prefixation in $50 \mathrm{mM}$ phosphate buffer, $\mathrm{pH}$ $7,1.5 \%$ formaldehyde, and $0.05 \%$ Triton X-100; fixation in $75 \mathrm{mM}$ phosphate buffer, $2 \%$ paraformaldehyde, and $0.5 \%$ glutaraldehyde) embedded in Technovit 7100 resin (Kulzer).

Tissue localization by in situ PCR was carried out as previously described ${ }^{57,58}$ with slight modifications. In brief, fresh roots of 14-day old rice plants were fixed in a solution containing $63 \%$ ethanol, $5 \%$ acetic acid and $2 \%$ formalin at $4{ }^{\circ} \mathrm{C}$ for $12 \mathrm{~h}$ and embedded in $5 \%$ agarose. Mature zone sections (thickness: 60 $\mu \mathrm{m}$ ) were treated with $2.5 \%$ DNase at $37^{\circ} \mathrm{C}$ for $45 \mathrm{~min}$. Reverse transcription reaction was then immediately performed in $1 \times \mathrm{RT}$ mix (Thermoscript RT) in presence of the OsK2.2-specific reverse primer (OsK2.2-qR) or in absence of OsK2.2 primer (mock control). The cDNA amplification was subsequently performed with gene-specific primers (OsK2.2-qF/qR) by PCR to integrate digoxigenin-11-dUTP (Roche; 
$4 \mu \mathrm{M}$ ), and sections were then blocked in $1 \mathrm{x}$ block buffer (1 mg BSA in $1 \mathrm{~mL} 1 \mathrm{x}$ PBS) at room temperature for 30 min prior to incubation with 1.5 units AP-conjugated anti-DIG Fab fragments (Roche) in 1x block buffer. A brief rinse in $1 x$ wash buffer $(0.1 \mathrm{M}$ Tris-Cl; $0.15 \mathrm{M} \mathrm{NaCl}, \mathrm{pH} 9.5)$ preceded an incubation until staining emergence in $50 \mu \mathrm{l} \mathrm{BM}$ purple (Roche). Finally, the processed tissue sections were rinsed with $1 \mathrm{x}$ wash buffer and then mounted in $40 \%$ glycerol on glass slide for visualization by optical microscopy.

\section{Plant experiments}

Nucleotide deletions in two independent CRISPR-Cas9mutant lines were ascertained by sequencing (Supplementary Fig. 2a). The expression level of OsK2.2 in OE lines (Supplementary Fig. 2b) was determined by RT-qPCR relative to the transcript abundance of OsActin (primers: see Supplementary Table 2). For both the KO mutant and $O E$ lines, homozygous plants were used: T3 generation in the experiments performed with plants grown in hydroponics conditions, and T3 and T4 generations in the two successive field test experiments.

To obtain relatively uniform starting materials without significant biomass difference between the plants (KO mutant and $O E$ lines, with respect to the respective wildtype plants), seedlings were initially grown in a growth chamber for 10 or 14 days (depending on the experiment) in IRRI nutrient solution containing 1 $\mathrm{mM} \mathrm{K}^{+}$as previously described ${ }^{59}$. Then, when the external concentration of $\mathrm{K}^{+}$was to be varied (modified IRRI solution), $\mathrm{KH}_{2} \mathrm{PO}_{4}$ and $\mathrm{K}_{2} \mathrm{SO}_{4}$ were replaced by $\mathrm{NaH}_{2} \mathrm{PO}_{4}$ and $\mathrm{Na}_{2} \mathrm{SO}_{4}$, respectively, and $\mathrm{KCl}$ was used as the sole $\mathrm{K}^{+}$source.

For the collection of sap exudates, 14-day-old rice seedlings were grown for 3 further days in absence of $\mathrm{K}^{+}$and then re-supplemented with $20 \mathrm{mM} \mathrm{K}^{+} 3 \mathrm{~h}$ before sap sample collection. Shoots were excised $3 \mathrm{~cm}$ above the basal root-shoot junction. The phloem exudates were collected in $5 \mathrm{ml}$ of $20 \mathrm{mM}$ EDTA for $3 \mathrm{~h}$ from uniformly excised shoots (the excised tissues being bathed in the EDTA solution) using the method described previously ${ }^{60-64}$. The xylem sap was collected from the excised stumps for $3 \mathrm{~h}$ with absorbent cotton according to Gao's method ${ }^{65}$.

A similar plant growth protocol was used to obtain plants for photosynthetic parameter measurements. The experiments were performed on the second last leaf using a Li-Cor 6400 gas exchange portable photosynthesis system (Li-Cor, Lincoln, Nebraska, USA) $2 \mathrm{~h}$ after the supplementation with $20 \mathrm{mM} \mathrm{K}^{+}$and were completed within $2 \mathrm{~h}$.

To investigate the contribution of OsK2.2 to plant growth or to plant adaptation to saline conditions, 10day-old seedlings were grown for 7 further days in $10 \mathrm{mM} \mathrm{K}^{+}$to observe growth phenotypes, or for 14 further days in $100 \mathrm{mM} \mathrm{NaCl}$ to check salt tolerance.

Local net $\mathrm{K}^{+}$influxes into roots of 10-day-old intact plants were measured using the non-invasive microtest technique (NMT) (Xuyue Science and Technology Company, Beijing, China). The external solution 
bathing the roots contained $1 \mathrm{mM} \mathrm{K}^{+}$.

Plant samples were digested with $\mathrm{H}_{2} \mathrm{SO}_{4}-\mathrm{H}_{2} \mathrm{O}_{2}$ for $\mathrm{N}, \mathrm{K}^{+}$and $\mathrm{Na}^{+}$content measurements. $\mathrm{N}$ was assayed by using an elemental analyzer (Westco). $\mathrm{K}^{+}$and $\mathrm{Na}^{+}$were assayed in the digestion solutions and in the collected sap samples by flame spectrophotometry.

\section{Field experiments}

Field experiments were carried out during 2 seasons (in 2017 and 2019) in a typical paddy soil. The initial soil $\mathrm{pH}$ was 5.2. Available $\mathrm{N}, \mathrm{P}_{2} \mathrm{O}_{5}$ and $\mathrm{K}_{2} \mathrm{O}$ was $7.3,7.0$ and $70 \mathrm{ppm}$, respectively. For each testing seasons, fertilizers were applied at the following amounts (per hm²): $200 \mathrm{~kg}$ nitrogen, $90 \mathrm{~kg} \mathrm{P}_{2} \mathrm{O}_{5}$ and 240 $\mathrm{kg} \mathrm{K}_{2} \mathrm{O}$. Rice seedlings were planted at a density of $0.2 \times 0.25(\mathrm{~m} \times \mathrm{m})$ per plant. Agronomic traits were observed at the late grain-filling stage. Flag leaf contents in $\mathrm{K}^{+}$and $\mathrm{N}$ were also measured at this stage.

\section{Electrophysiology in oocytes}

Healthy oocytes were obtained from mature Xenopus laevis frogs and prepared as previously reported $^{66,67}$. The cRNAs of OSCBL 1 and OSCIPK23 were transcribed in vitro using the T7 mMESSAGEmMACHINE® Kit (AM1344, Thermo Fisher Scientific). Oocytes were micro-injected (Nanoliter 2000, WPI, Sarasota, FL, USA) with 60 ng plasmid DNA ${ }^{25,67}$ containing the sequence encoding the OsK2.2 wild-type channel or a derived chimeric channel. For co-expression with the CBL1/CIPK23 complex, the channel plasmid DNA (60 ng) was injected together with $30 \mathrm{ng}$ cRNA of OSCBL 1 and of OSCIPK23. Oocytes injected with the same volume and/or amount of $\mathrm{H}_{2} \mathrm{O}$ and/or kinase cRNAs only were used as controls. Injected oocytes were incubated at $19^{\circ} \mathrm{C}$ in ND96 solution (mM: $96 \mathrm{NaCl}, 2 \mathrm{KCl}, 1 \mathrm{MgCl}_{2}, 1.8$ $\mathrm{CaCl}_{2}, 5$ HEPES, pH 7.4, supplemented with $0.05 \mathrm{mg} / \mathrm{mL}$ gentamycin) for $48 \mathrm{~h}$ before the two-electrode voltage-clamp measurements using an Axoclamp 900A amplifier (Molecular Devices, San Jose, CA, USA). The bath solutions contained $1.8 \mathrm{mM} \mathrm{CaCl}_{2}, 1 \mathrm{mM} \mathrm{MgCl}_{2}, 5 \mathrm{mM}$ HEPES, pH 7.4, and the desired concentration of $\mathrm{KCl}, \mathrm{NaCl}$ being added to the solution so that the total concentration of monovalent $\mathrm{Na}^{+}$ $+\mathrm{K}^{+}$was $100 \mathrm{mM}$. The oocytes were clamped to voltage steps ranging from $-160 \mathrm{mV}$ to $+20 \mathrm{mV}$ with a $+10 \mathrm{mV}$ increment (the holding voltage being $-40 \mathrm{mV}$ ).

\section{Fluorescence intensity determination}

The oocyte preparation, plasmid injection and incubation were performed as for the electrophysiological experiments. The GFP fluorescence was observed using a Laser scanning confocal microscope (LSM710, 
Zeiss) $48-60 \mathrm{~h}$ after injection. Fluorescence intensity of the oocyte membrane was quantified from at least 8 cells with the "ZEN 2009 Light Edition" software provided by the microscope setup.

\section{Data analysis}

The electrophysiological data were analyzed using Clampfit 10.3 (Molecular Devices). The statistical analysis and graphing were performed with SigmaPlot 12.5 and GraphPad Prism 8.0.2 softwares. Data were expressed as means \pm SE. Levels of statistical significance were analyzed by Student's t-test. The symbols * and ** indicate significances at $p<0.05$ and $p<0.01$, respectively.

\section{Data availability}

The data that support the findings of this study are available within the paper and its Supplementary Information or from the corresponding authors upon request.

\section{Declarations}

\section{Acknowledgements}

This work was supported by the National Natural Science Foundation of China (31672230), the National Key Research and Development Program of China (2016YFD0100703/2016YFD0100700), a doctoral fellowship from the French Embassy in Vietnam (to T.H.N.), an ANR-DFG grant (ANR-20-CE92-0005 to A.A.V.) and an ANR IA grant (ANR-11-RSNR-0005 to E.G. and H.S.). We thank Rong Huang and Zuohao $\mathrm{Ma}$, Analytical and Testing Center, Institute of Soil Science, Chinese Academy of Sciences and Dr. Shuwei Wang, State Experimental Station of Agro-Ecosystem in Changshu, Chinese Academy of Sciences, for technical assistance.

\section{Author contributions}

Y.S. and A.-A.V. conceived and designed the research with the help of H.S. T.-H.N., S.Y., S.W., A.V. and E.G. contributed to the localization analysis. S.Y. designed and screened the mutant and overexpression rice lines and carried out phenotypic analyses. S.Y., T.-H.N. and J.L. did the electrophysiological experiments. CF did the phylogenetic analyses. S.W., Y.H., D. H. and J.W. contributed to field experiments. S.Y. analyzed the data. H.S., Y.S., A.-A.V. and S.Y. wrote the paper. The authors would dedicate this paper to celebrate Dr. Hervé Sentenac's 30 years of efforts to advance the knowledge of plant potassium channels. 


\section{Competing interests}

The authors declare no competing interests.

\section{Additional information}

Supplementary information is available for this paper.

\section{References}

1. Marschner, P. Marschner's Mineral nutrition of higher plants (3rd Edition): In Functions of macronutrients (San Diego: Academic Press, 2011).

2. Nieves-Cordones, M., Al Shiblawi, F. R. \& Sentenac, H. Metal Ions in Life Sciences, the alkali metal ions, their role for life: Roles and transport of sodium and potassium in plants (Springer, 2016)

3. Mäser, P. et al. Phylogenetic relationships within cation transporter families of Arabidopsis. Plant Physiol. 126, 1646-1667 (2001).

4. Véry, A.-A. et al. Molecular biology of $\mathrm{K}^{+}$transport across the plant cell membrane: what do we learn from comparison between plant species? J. Plant Physiol.171, 748-769 (2014).

5. Nakamura, R. L.et al. Expression of an Arabidopsis potassium channel gene in guard cells. Plant Physiol. 109, 371-374 (1995).

6. Pilot, G. et al. Guard cell inward $\mathrm{K}^{+}$channel activity in Arabidopsis involves expression of the twin channel subunits KAT1 and KAT2. J. Biol. Chem. 276, 3215-3221 (2001).

7. Hosy, E. et al. The Arabidopsis outward $\mathrm{K}^{+}$channel GORK is involved in regulation of stomatal movements and plant transpiration. Proc. Natl. Acad. Sci. USA 100, 5549-5554 (2003).

8. Lebaudy, A. et al. Plant adaptation to fluctuating environment and biomass production are strongly dependent on guard cell potassium channels. Proc. Natl. Acad. Sci. USA 105, 5271-5276 (2008).

9. Lacombe, B. et al. A shaker-like $\mathrm{K}^{+}$channel with weak rectification is expressed in both source and sink phloem tissues of Arabidopsis. Plant Cell 12,837-851 (2000).

10. Marten, I. et al. AKT3, a phloem-localized $\mathrm{K}^{+}$channel, is blocked by protons. Proc. Natl. Acad. Sci. USA 96, 7581-7586 (1999).

11. Huang, Y. N. et al. The rectification control and physiological relevance of potassium channel OsAKT2. Plant Physiol. 187, 2296-2310 (2021).

12. Sentenac, $\mathrm{H}$. et al. Cloning and expression in yeast of a plant potassium ion transport system. Science 256, 663-665 (1992).

13. Hirsch, R. E., Lewis, B. D., Spalding, E. P. \& Sussman, M. R. A role for the AKT1 potassium channel in plant nutrition. Science 280, 918-921(1998). 
14. Gaymard, F. et al. Identification and disruption of a plant shaker-like outward channel involved in $\mathrm{K}^{+}$ release into the xylem sap. Cell 94, 647-655 (1998).

15. Pilot, G., Pratelli, R., Gaymard, F., Meyer, Y. \& Sentenac, H. Five-group distribution of the Shaker-like $K^{+}$ channel family in higher plants. J. Mol. Evol. 56, 418-434 (2003).

16. $\mathrm{Li}$, J. et al. The Os-AKT1 channel is critical for $\mathrm{K}^{+}$uptake in rice roots and is modulated by the rice CBL1-CIPK23 complex. Plant cell 26, 3387-3402 (2014).

17. Nguyen, T. H. et al. A dual role for the OsK5.2 ion channel in stomatal movements and $\mathrm{K}^{+}$loading into xylem sap. Plant Physiol. 174, 2409-2418 (2017).

18. Müller-Röber, B. et al. Cloning and electrophysiological analysis of KST1, an inward rectifying $\mathrm{K}^{+}$ channel expressed in potato guard cells. EMBO J. 14, 2409-2416 (1995).

19. Pratelli, R. et al. A grapevine gene encoding a guard cell $\mathrm{K}^{+}$channel displays developmental regulation in the grapevine berry. Plant Physiol. 128, 564-577 (2002).

20. Cuéllar, T. et al. Potassium transport in developing fleshy fruits: the grapevine inward $\mathrm{K}^{+}$channel VvK1.2 is activated by CIPK-CBL complexes and induced in ripening berry flesh cells. Plant J. 73, 1006-1018 (2013).

21. Büchsenschütz, K. et al. Differential expression of $\mathrm{K}^{+}$channels between guard cells and subsidiary cells within the maize stomatal complex. Planta 222, 968-976 (2005).

22. Su, Y. H. et al. Regulation by external $\mathrm{K}^{+}$in a maize inward shaker channel targets transport activity in the high concentration range. Plant Cell 17, 1532-1548 (2005).

23. Boscari, A. et al. Potassium channels in barley: cloning, functional characterization and expression analyses in relation to leaf growth and development. Plant Cell \& Environ. 32, 1761-1777 (2009).

24. Langer, $\mathrm{K}$. et al. The poplar $\mathrm{K}^{+}$channel KPT1 is associated with $\mathrm{K}^{+}$uptake during stomatal opening and bud development. Plant J. 37, 828-838 (2004).

25. Yang, G. Z., Sentenac, H., Véry, A.-A., \& Su, Y. H. Complex interactions among residues within pore region determine the $\mathrm{K}^{+}$dependence of a KAT1-type potassium channel AmKAT1. Plant J. 83, 401412 (2015).

26. Li, J. L. et al. Functional identification of a GORK potassium channel from the ancient desert shrub Ammopiptanthus mongolicus (Maxim.) Cheng f. Plant Cell Rep. 35, 803-815 (2016).

27. Geiger, D. et al. Heteromeric AtKC1 AKT1 channels in Arabidopsis roots facilitate growth under $\mathrm{K}^{+}$limiting conditions. J. Biol. Chem. 284, 21288-21295 (2009).

28. Jeanguenin, L. et al. AtKC1 is a general modulator of Arabidopsis inward Shaker channel activity. Plant J. 67, 570-582 (2011).

29. Véry, A.-A. \& Sentenac, H. Molecular mechanisms and regulation of $\mathrm{K}^{+}$transport in higher plants. Annu. Rev. Plant Biol. 54, 575-603 (2003).

30. Ehrhardt, T., Zimmermann, S. \& Müller-Röber, B. Association of plant $\mathrm{K}^{+}$in channels is mediated by conserved C termini and does not affect subunit assembly. FEBS Lett. 409, 166-170 (1997). 
31. Daram, P., Urbach, S., Gaymard, F., Sentenac, H. \& Chérel, I. Tetramerization of the AKT1 plant potassium channel involves its C-terminal cytoplasmic domain. EMBO J. 16, 3455-3463 (1997).

32. $\mathrm{Xu}$, J. et al. A protein kinase, interacting with two calcineurin $\mathrm{B}-$ like proteins, regulates $\mathrm{K}^{+}$transporter AKT1 in Arabidopsis. Cell 125, 1347-1360 (2006).

33. Saito, S., \& Uozumi, N. Calcium-regulated phosphorylation systems controlling uptake and balance of plant nutrients. Front. Plant Sci. 11, 44 (2020).

34. Sánchez-Barrena, M. J. et al. Recognition and activation of the plant AKT1 potassium channel by the kinase CIPK23. Plant Physiol. 182, 2143-2153 (2020).

35. Tang, R. J., Wang, C., Li, K. \& Luan, S. The CBL-CIPK calcium signaling network: unified paradigm from 20 years of discoveries. Trends Plant Sci. 25, 604-617 (2020).

36. Thiruppathi, D. Molecular snap shots of the AKT1-CIPK23 complex involved in $\mathrm{K}^{+}$uptake. Plant Physiol. 182, 1814-1815 (2020).

37. Golldack, D., Quigley, F., Michalowski, C. B., Kamasani, U. R., \& Bohnert, H.J. Salinity stress-tolerant and-sensitive rice (Oryza sativa L.) regulate AKT1-type potassium channel transcripts differently. Plant Molecular Biology 51, 71-81 (2003).

38. Lee, S.C. et al. A protein phosphorylation/dephosphorylation network regulates a plant potassium channel. Proc. Natl. Acad. Sci. USA 104, 15959-15964 (2007).

39. Nieves-Cordones, M., Alemán, F., Martínez, V. \& Rubio, F. K+ uptake in plant roots. The systems involved their regulation and parallels in other organisms. J. Plant Physiol. 171, 688-695 (2014).

40. Santa-Maria, G. E., Oliferuk, S. \& Moriconi J. I. KT-HAK-KUP transporters in major terrestrial photosynthetic organisms: A twenty years tale. J. Plant Physiol. 226, 77-90 (2018).

41. Altschul, S. F. \& Lipman, D. J. Protein database searches for multiple alignments. Proc. Natl. Acad. Sci. USA 87, 5509-5513 (1990).

42. de Castro, E. et al. ScanProsite: detection of PROSITE signature matches and ProRule-associated functional and structural residues in proteins. Nucleic Acids Res. 34(Web Server issue), W362-W365 (2006).

43. Marchler-Bauer, A., \& Bryant, S. H. CD-Search: protein domain annotations on the fly. Nucleic Acids Res. 32(Web Server issue), W327-W331 (2004).

44. Véry, A.-A., Gaymard, F., Bosseux, C., Sentenac, H. \& Thibaud, J. B. Expression of a cloned plant K ${ }^{+}$ channel in Xenopus oocytes: analysis of macroscopic currents. Plant J. 7, 321-332 (1995).

45. Xicluna, J. et al. Increased functional diversity of plant $\mathrm{K}^{+}$channels by preferential heteromerization of the shaker-like subunits AKT2 and KAT2. J. Biol. Chem. 282, 486-494 (2007).

46. Hedrich, R. Ion channels in plants. Physiol. Rev. 92, 1777-1811 (2012).

47. Ragel, P., Raddatz, N., Leidi, E. O., Quintero, F. J. \& Pardo J. M. Regulation of $\mathrm{K}^{+}$Nutrition in Plants. Front Plant Sci. 10, 28 (2019). 
48. Han, M., Wu, W., Wu, W.H. \& Wang Y. Potassium transporter KUP7 is involved in $\mathrm{K}^{+}$acquisition and translocation in Arabidopsis root under $\mathrm{K}^{+}$-limited conditions. Mol. Plant 9, 437-446 (2016).

49. Chen, G. et al. Rice potassium transporter OsHAK1 is essential for maintaining potassium-mediated growth and functions in salt tolerance over low and high potassium concentration ranges. Plant, Cell \& Environ. 38, 2747-2765 (2015).

50. Yang, T.Y. et al. The role of a potassium transporter OsHAK5 in potassium acquisition and transport from roots to shoots in rice at low potassium supply levels. Plant Physiol. 166, 945-959 (2014).

51. Shen, Y. et al. The potassium transporter OsHAK21 functions in the maintenance of ion homeostasis and tolerance to salt stress in rice. Plant, Cell \& Environ. 38, 2766-2779 (2015).

52. Miao, J. et al. Targeted mutagenesis in rice using CRISPR-Cas system. Cell Res. 23, 1233-1236 (2013).

53. Hu, X. X. et al. Expanding the range of CRISPR/Cas9 genome editing in Rice. Mol. Plant 9, 943-945 (2016).

54. Ma, X. L. et al. A robust CRISPR/Cas9 system for convenient high-efficiency multiplex genome editing in monocot and dicot Plants. Mol. Plant 8, 1274-1284 (2015).

55. Wang, Z. et al. A practical vector for efficient knockdown of gene expression in rice (Oryza sativa L.). Plant Mol. Biol. Rep. 22, 409-417(2004).

56. Jabnoune, M. et al. Diversity in expression patterns and functional properties in the rice HKT transporter family. Plant Physiol. 150, 1955-1971 (2009).

57. Athman, A. et al. Protocol: a fast and simple in situ PCR method for localising gene expression in plant tissue. Plant Methods 10, 29 (2014).

58. Przybecki, Z., Siedlecka, E., Filipecki, M. \& Urbanczyk-Wochniak, E. In Situ reverse transcription PCR on plant tissues. Methods Mol. Biol. 18, 181-198 (2006).

59. Yang, S. Y. et al. Internal ammonium excess induces ROS-mediated reactions and causes carbon scarcity in rice. BMC Plant Biol. 20, 143 (2020).

60. Killiny, N. Collection of the phloem sap, pros and cons. Plant Signal. Behav. 14, e1618181 (2019).

61. Du, B. et al. Phloem-exudate proteome analysis of response to insect brown pant-hopper in rice. J. Plant Physiol. 183, 13-22 (2015).

62. Tetyuk, O., Benning, U. F. \& Hoffmann-Benning, S. Collection and analysis of Arabidopsis phloem exudates using the EDTA-facilitated method. J. Vis. Exp. 80, e51111 (2013).

63. Urquhart, A. A. \& Joy, K. W. Use of phloem exudate technique in the study of amino acid transport in pea plants. Plant Physiol. 68, 750-754 (1981).

64. King, R. W. \& Zeevaart, J. A. D. Enhancement of phloem exudation from cut petioles by chelating agents. Plant Physiol. 53, 96-103 (1974).

65. Gao, Y. X. et al. Ammonium nutrition increases water absorption in rice seedlings (Oryza sativa L.) under water stress. Plant Soil 331, 193-201 (2010). 
66. Hao, D. L. et al. Functional characterization of the Arabidopsis ammonium transporter AtAMT1;3 with the emphasis on structural determinants of substrate binding and permeation properties. Front. Plant Sci. 11, 571 (2020).

67. Wang, L. et al. The S1-S2 linker determines the distinct pH sensitivity between ZmK2.1 and KAT1. Plant J. 85, 675-685 (2016).

68. Edgar, R. C. MUSCLE: multiple sequence alignment with high accuracy and high throughput. Nucleic Acids Res. 32, 1792-1797 (2004).

69. Castresana, J. Selection of conserved blocks from multiple alignments for their use in phylogenetic analysis. Mol. Biol. Evol. 17, 540-552 (2000).

70. Gouy, M., Guindon, S. \& Gascuel, O. SeaView version 4: A multiplatform graphical user interface for sequence alignment and phylogenetic tree building. Mol. Biol. Evol. 27, 221-224 (2010).

71. Guindon, S. et al. New algorithms and methods to estimate maximum-likelihood phylogenies: assessing the performance of PhyML 3.0. Syst. Biol. 59, 307-321 (2010).

\section{Figures}




\section{Fig. 1}

a

\section{OsK2.2}

Hydrophobic core

S1 $1{ }^{54} 56$
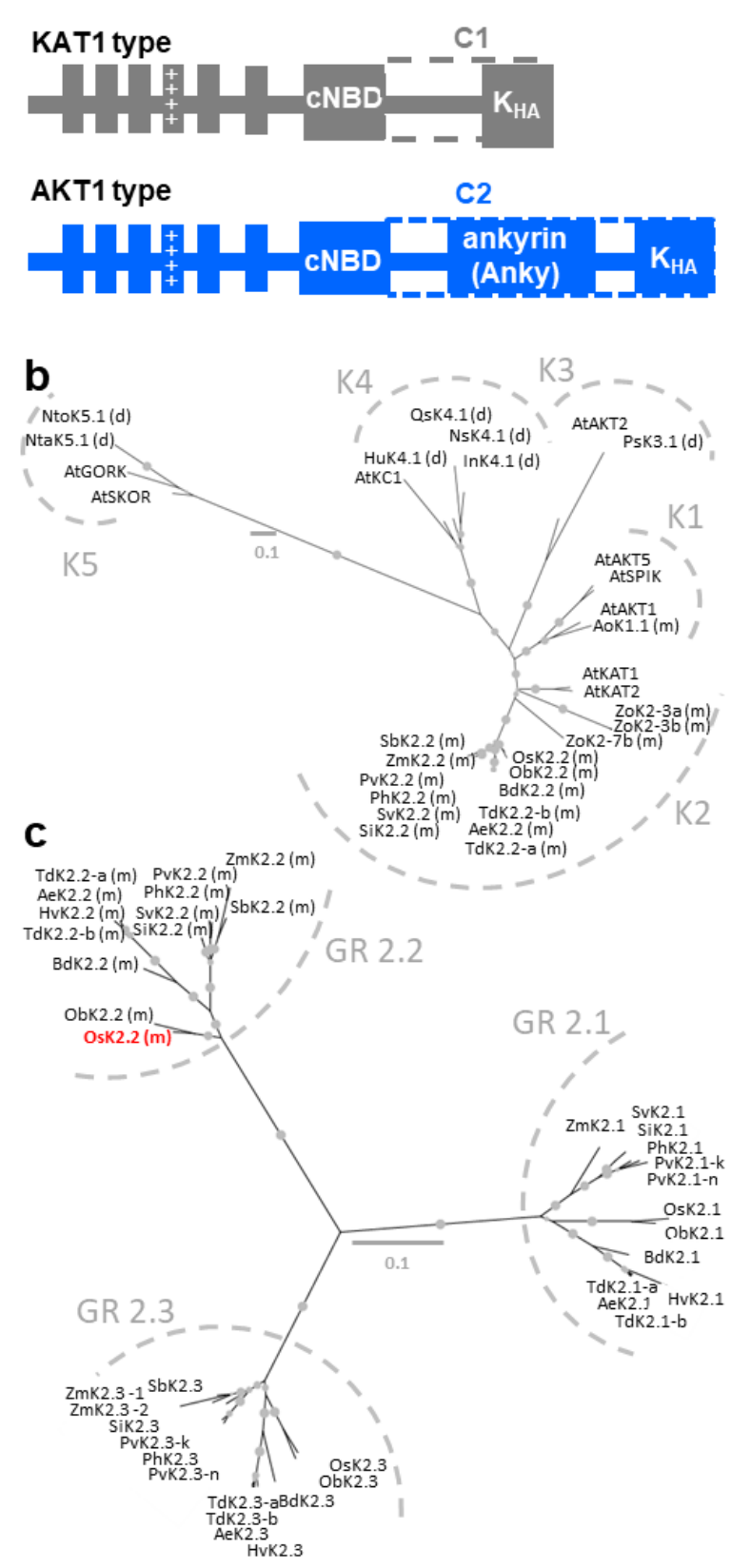

Figure 1

OsK2.2 is representative of short Shaker channels especially found in Poales.

a, Schematic representation of the predicted structure of OsK2.2 in comparison to that of the Arabidopsis AtKAT1 and the rice OsAKT1. Shaker channels display a hydrophobic core composed of 6 transmembrane segments, named S1 to S6 (represented by the 6 vertical bars in the drawings). S4 
contains positively charged amino acids and behaves as a voltage sensor, rendering the channel sensitive to voltage. A putative cyclic nucleotide binding domain (CNBD) is present downstream of the channel transmembrane domain. In both AtKAT1 and OsAKT1, the C-terminal region downstream of the cNBD contains the so-called $\mathrm{K}_{\mathrm{HA}}$ domain, rich in hydrophobic and acidic residues and proposed to allow channel clustering in the membrane ${ }^{30}$. In OsAKT1, an ankyrin (Anky) domain allowing interactions with regulatory proteins is present between the $\mathrm{CNBD}$ and $\mathrm{K}_{\mathrm{HA}}$ domains. These $\mathrm{C}$-terminal regions downstream of the cNBD, present in AtKAT1 and OsAKT1, but not in OsK2.2, have been named C1 and C2, respectively, in the present report.

b, Phylogenetic relationships between the nine Shaker channel proteins present in Arabidopsis and innate "short" Shaker polypeptides encoded by genes whose full length open reading frame gives rise to a polypeptide displaying a C-terminal region devoid of $\mathrm{K}_{\mathrm{HA}}$ and Anky domains. The short Shakers were identified in silico, from a set of 1920 sequences belonging to the Viridae Shaker family recovered using BLASTP (see main text). A "(d)" or a "(m)" following the name of the short channel protein indicates that the corresponding plant species is a dicot or a monocot, respectively. The letter $K$ followed by $\# 1,2,3,4$ or 5 indicates that the corresponding channel belongs to the Shaker family $\# 1,2,3,4$ or 5 , respectively, according to the nomenclature proposed by Pilot ${ }^{15}$. Selected Shakers sequences were aligned with Muscle(v3.8.31 ${ }^{68}$. Conserved blocks of sequences were selected with GBlocks $(0.91 b)^{69}$ in Seaview ${ }^{70}$. PhyML (version 3.3.3:3.3.20190909-1) ${ }^{71}$ in Seaview was employed to construct a maximum likelihood unrooted phylogenetic tree. Bootstrapping was performed with 1000 replicates. The phylogenetic tree was drawn with iTOL (https://itol.embl.de/upload.cgi). Bootstrap values are represented as grey circles with diameter proportional to the value, drawn at the corresponding nodes. The scale bar corresponds to a distance of 10 changes per 100 amino acid positions.

c, Phylogenetic relationships of all Shaker polypeptides belonging to the Shaker subfamily \#2 from Poales species identified as possessing a gene encoding a "short" Shaker sequence (i.e., all identified subfamily \#2 short Shakers from plants except those from Zingiber officinale). The plant species taken into account are the 11ones (including rice) identified in Panel B and barley (see text). The unrooted phylogenetic tree, performed as in $\mathbf{b}$, identifies 3 groups, GR2.1 to GR2.3. GR2.2 comprises all the polypeptides displaying a short C-terminal region and only channels of this type. The scale bar corresponds to a distance of 10 changes per 100 amino acid positions.

Ae: Aegilops tauschii; Ao: Asparagus officinalis; At: Arabidopsis thaliana; Bd: Brachypodium distachyon; Hu: Herrania umbratica; Hv: Hordeum vulgare, In: Ipomoea nil; Ns: Nicotiana sy/vestris; Nta: Nicotiana tabacum; Nto: Nicotiana tomentosiformis; Ob: Oryza brachyantha; Os: Oryza sativa; Ph: Panicum hallii; Ps: Papaver somniferum, Pv: Panicum virgatum; Qs: Quercus suber, Sb: Sorghum bicolor, Si: Setaria italica; Sv: Setaria viridis, Td: Triticum dicoccoides; Zm: Zea mays; Zo: Zingiber officinale. Accession references are listed in Supplementary Table 1. 
Fig. 2

a
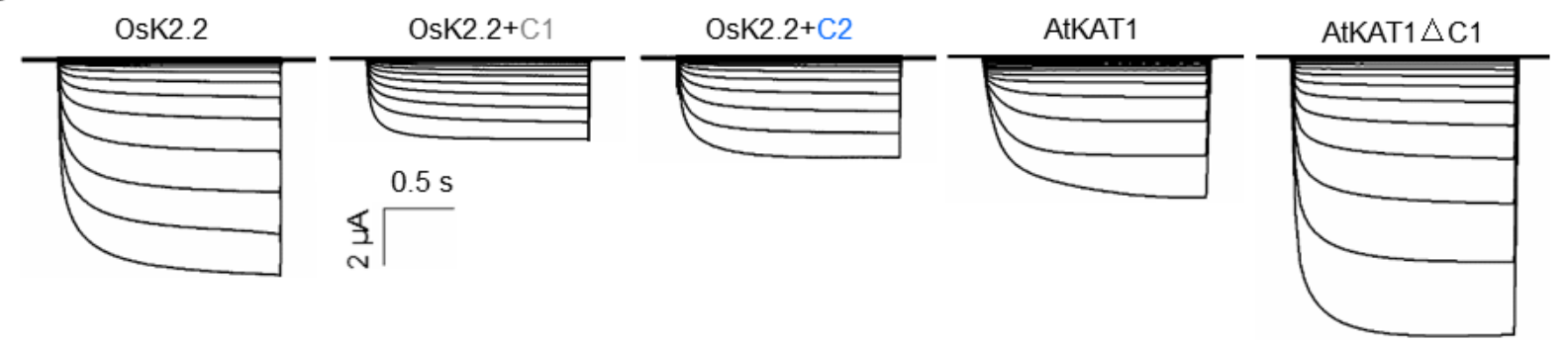

b
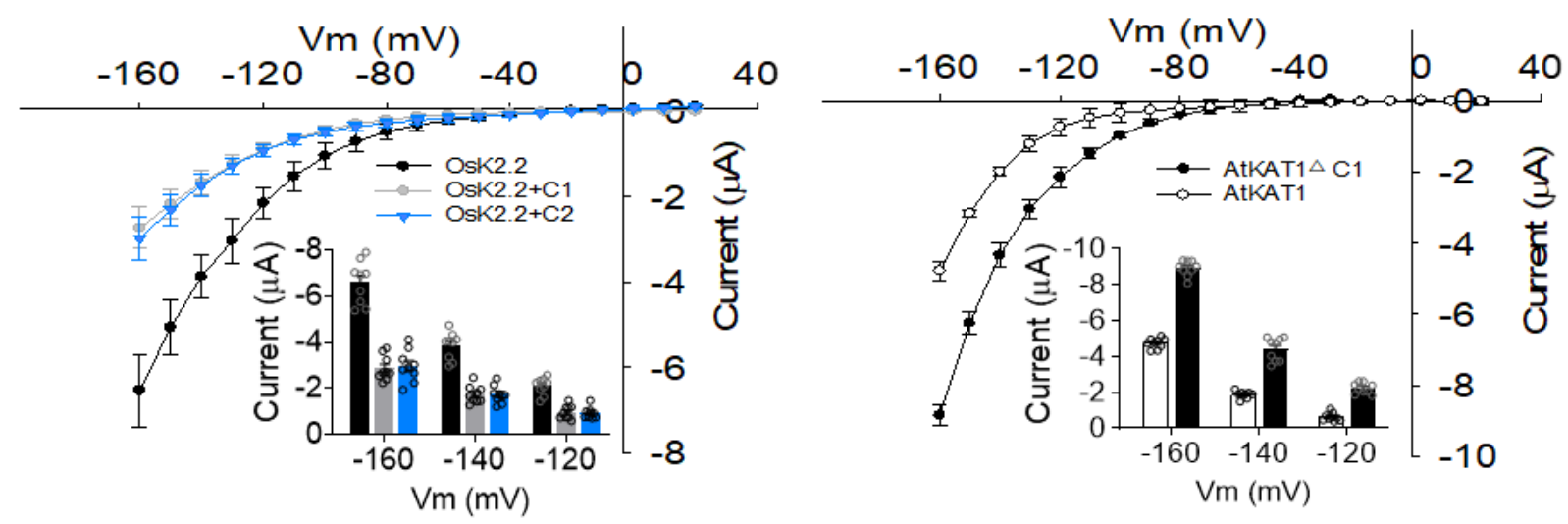

C
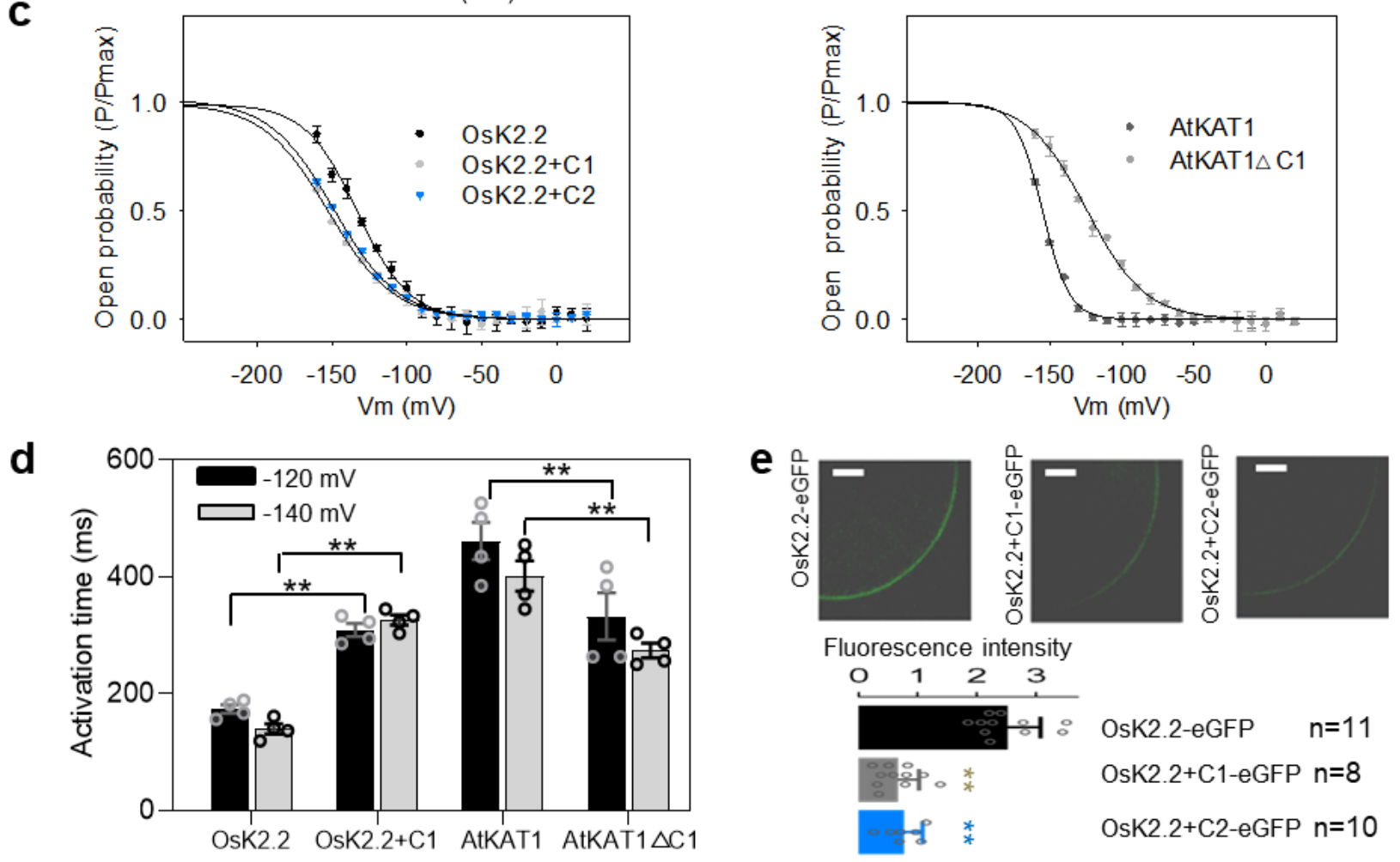

Fluorescence intensity

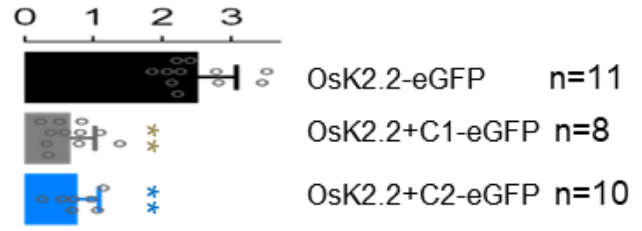

Figure 2

Functional characterization in Xenopus oocytes of OsK2.2 and related channel constructs.

a, Representative recordings obtained in an oocyte expressing either OsK2.2, or a chimeric construct fusing OsK2.2 to either the C-terminal region $\mathrm{C} 1$ from AtKAT1 or C2 from OsAKT1 (OsK2.2+C1 and OsK2.2+C2, respectively; see Fig. 1a), or AtKAT1 or AtKAT1 deleted from its C1 region (AtKAT1 $\triangle \mathrm{C} 1$ ). The 
recording protocol was composed of 1.5-second voltage steps ranging from - 160 to $+20 \mathrm{mV}$ with $10 \mathrm{mV}$ increments (holding potential: $-40 \mathrm{mV}$ ). The external solution bathing the oocytes contained $50 \mathrm{mM} \mathrm{K}^{+}$.

b, Current-voltage $\mathbb{Q} \mathrm{I} \vee \mathbb{V}$ relationships of the wildtype OsK2.2 and of the chimeric channelsOsK2.2+C1 and OsK2.2+C2 (left panel), and of AtKAT1 and the related shortened channelAtKAT1 $\triangle \mathrm{C} 1$ (right panel). Inset bar graphs: statistical analysis of the current amplitudes at $-160 \mathrm{mV},-140 \mathrm{mV}$ and $-120 \mathrm{mV}$, respectively. Means \pm SE $(n=9)$.

c, Gating properties. Voltage-dependence of the relative open probability (P/Pmax) of OsK2.2 and of the related chimeric channels OsK2.2+C1 and OsK2.2+C2 (left), and of AtKAT1 and the related shortened channelAtKAT1 $\triangle \mathrm{C} 1$ (right). Solid lines are Boltzmann fits to the data (as as previously described ${ }^{22,44}$. Means \pm SE $(n=4)$.

d, Channel activation time constants at -120 and $-140 \mathrm{mV}$. The time constants were obtained by fitting the current traces recorded at -120 and $-140 \mathrm{mV}$ to a single decaying exponential equation. Means $\pm \mathrm{SE}$ $(n=4)$.

e, Channel abundance at the oocyte cell membrane. The eGFP fluorescent protein was used to assess the channel abundance at oocyte cell membrane. It was fused to OsK2.2 and its related chimeras 0sK2.2+C1 and OsK2.2+C2. The resulting constructs were expressed in oocytes. Oocyte GFP fluorescence was observed using a laser scanning confocal microscope 48-60 hours after injection. Top: representative photographs of the green fluorescence visualized at the oocyte membrane (bar $=20 \mu \mathrm{m})$. Bottom: fluorescence intensity of the oocyte membrane quantified from at least 8 cells ("ZEN 2009 Light Edition" software of the confocal microscope). Data are means \pm SE. Statistical analysis: Student's $t$ tests. ${ }^{*}, P<$ $0.05 ; * \star, P<0.01$. 
Fig. 3
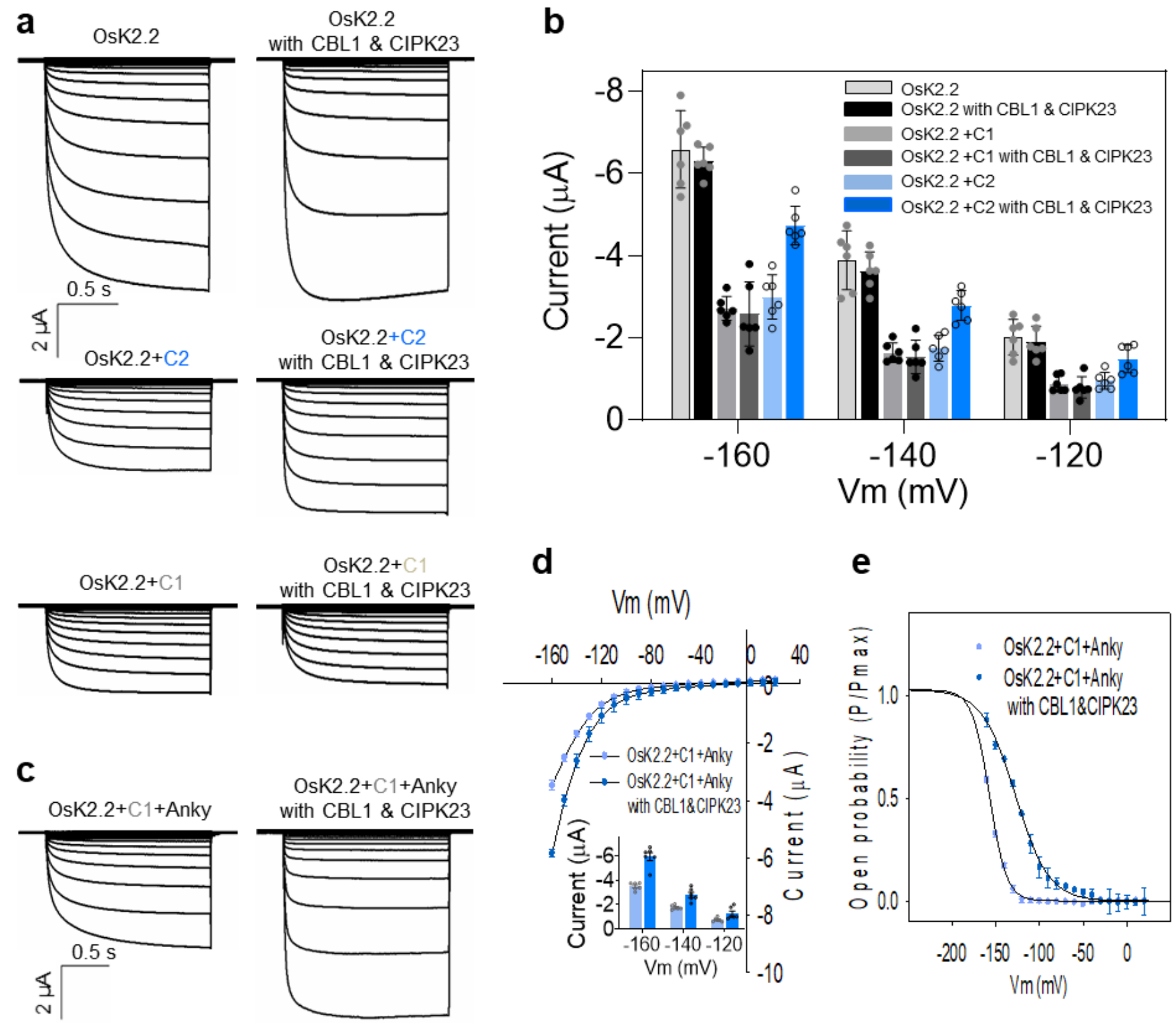

Figure 3

Absence of regulation of OsK2.2 channel activity in oocytes by the $\mathrm{Ca}^{2+}{ }^{2}$-sensing CBL1/CIPK23 kinase complex and responses of related chimeric channels to this complex.

OsK2.2 and OsK2.2-derived chimeric constructs were expressed alone or co-expressed with OsCBL1 and OsCIPK23 in oocytes. Electrophysiological recordings were performed in $50 \mathrm{mM}$ external $\mathrm{K}^{+}, \mathrm{pH}$ 7.4, using the same voltage-clamp protocol as in Fig. 2.

a,b, Representative current traces recorded in oocytes expressing either OsK2.2 or OsK2.2+C1 or OsK2.2+C2 (see Fig. 1a) alone or together with CBL1 and CIPK23 (A left and right panels, respectively) and statistical analysis of the currents recorded at $-120,-140$ and $-160 \mathrm{mV}$ in b. Means $\pm S E ; n=6$. 
c-e, The Anky domain present in the OsK2.2+C1+Anky chimeric construct renders the channel activity sensitive to the OsCBL1/OsCIPK23 complex. The OsK2.2+C1+ Anky construct results from the fusion of the OsAKT1 Anky domain (see Fig. 1a) downstream of $\mathrm{C} 1$ in the OsK2.2+C1 chimeric channel construct. c, Representative current traces recorded in an oocyte expressing OsK2.2+C1+Anky alone or together with CBL1 and CIPK23 (left and right panels, respectively). $\mathbf{d}$, Corresponding current-voltage relationships and statistical analysis (inset) of the currents recorded at $-120,-140$ and $-160 \mathrm{mV}$. Means $\pm \mathrm{SE} ; \mathrm{n}=6$. e, Gating properties in the absence or presence of the CBL1/CIPK23 complex. Voltage-dependence of the relative open probability (P/Pmax) analyzed using Boltzmann fits as in Fig. 2c. Means $\pm S E ; n=5$.

\section{Fig. 4}

a

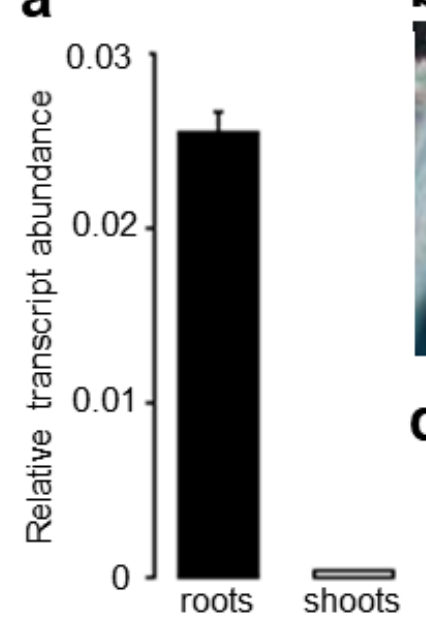

b
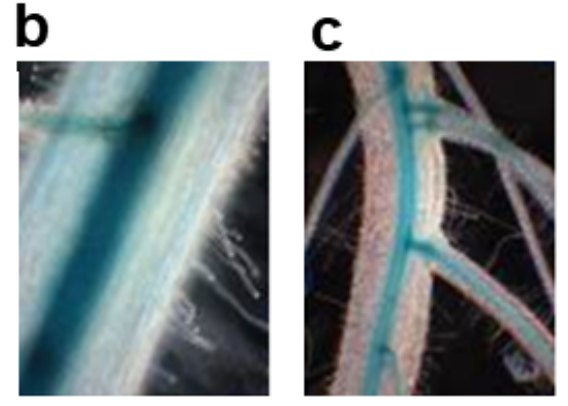

d
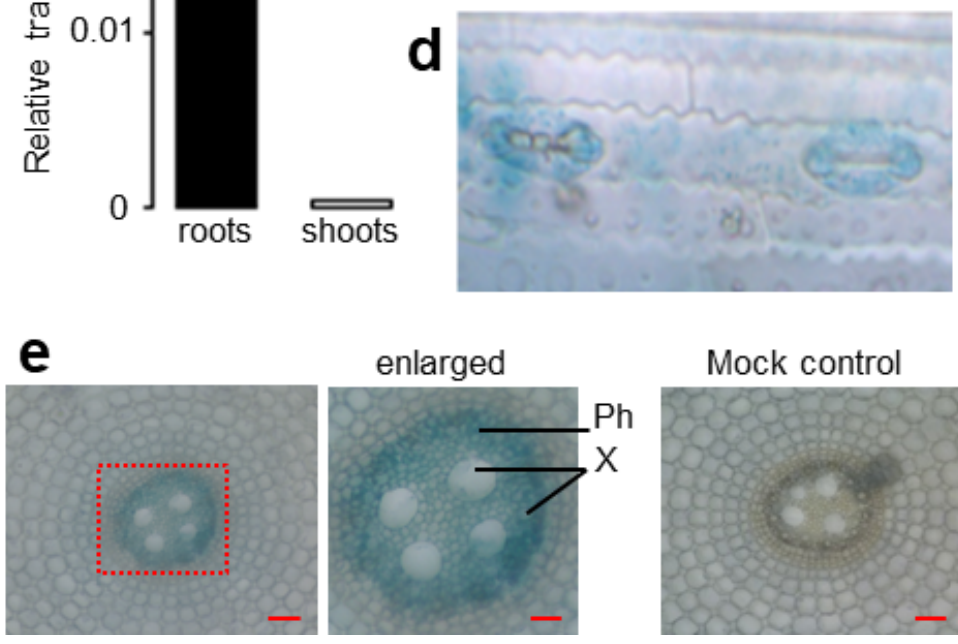

Mock control

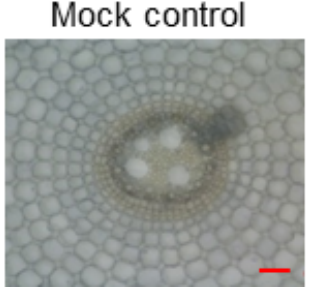

\section{Figure 4}

OsK2.2 expression in planta.

a. Expression level of OsK2.2 in roots and shoots. The expression level of OsK2.2 in 10-day-old rice seedlings was determined by quantitative RT-PCR. The transcript abundance was normalized using the OsActin $^{16}$ transcript level. Means \pm SE of three independent experiments.

a-c, Histochemical staining of GUS reporter activity driven by the OsK2.2 promoter. Representative photographs of GUS staining in the central cylinder of a seminal root (b), lateral root (c) and guard cells 
at leaf surface $(\mathbf{d})$.

e, In situ PCR localization of OsK2.2 expression in cross sections of mature zones of 10-day-old rice roots. Purple staining reveals the localization of OsK2.2 transcripts in phloem (Ph) and xylem (X) peripheral cells. Left panel: positive signal $(B a r=50 \mu \mathrm{m})$.Middle panel: magnification of the left panel (Bar=20 $\mu \mathrm{m}$ ). Right panel: negative (mock) control without OsK2.2-specific primer in the reverse transcription reaction (Bar $=50 \mu \mathrm{m})$.

\section{Fig. 5}
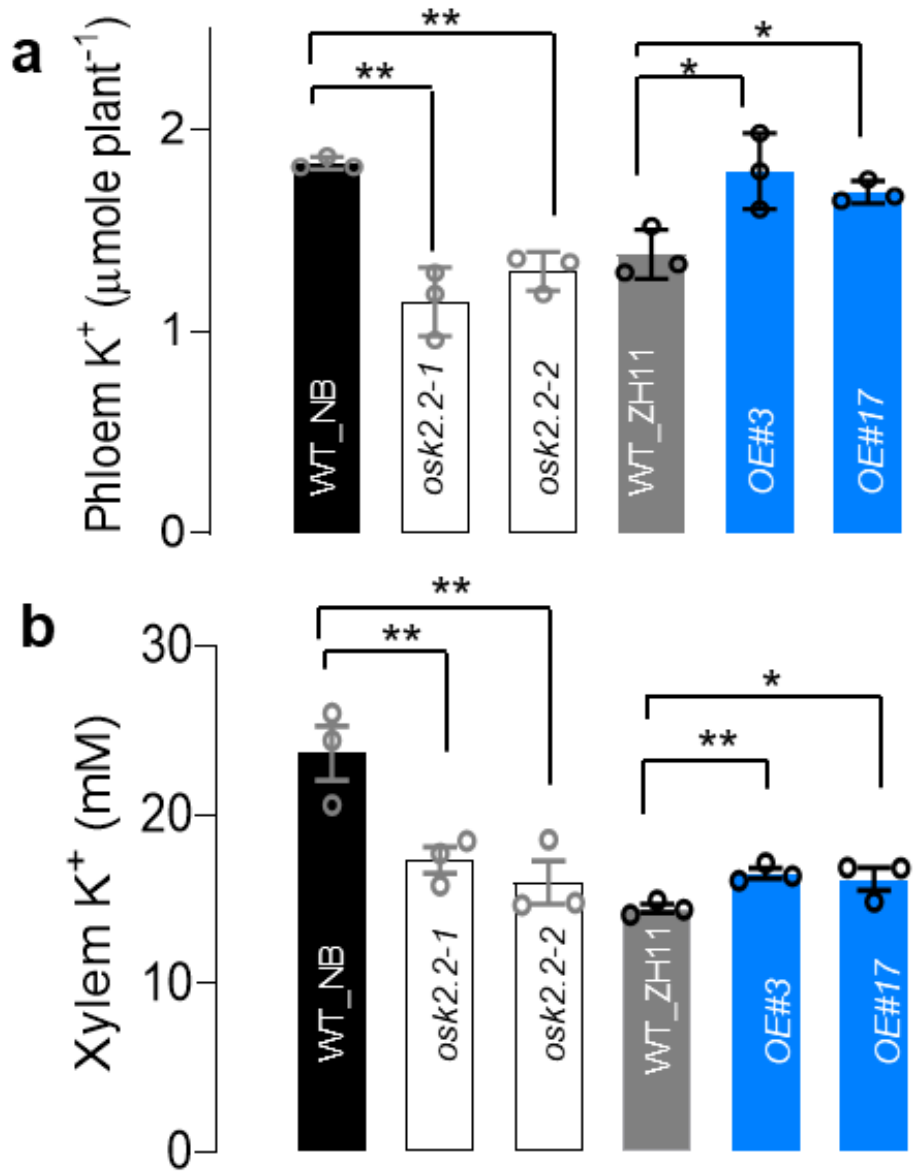

\section{Figure 5}

OsK2.2 plays a role in long distance $\mathrm{K}^{+}$transport via the phloem and $x y l e m$ saps.

Phloem and xylem sap samples were collected from two individual osk2.2 KO mutant line plants (osk2.21 and osk2.2-2) in Japonica Nipponbare genetic background (NB), from the corresponding control wild type NB plants (WT_NB), from two OsK2.2 overexpressing lines (OE\#3 and OE\#17) in Zhonghua-11 genetic background (ZH11) and from the corresponding control wild type ZH plants (WT_ZH11). The seedlings (T3-T4 homozygotes; 14-day-old) were submitted for 3 days to $\mathrm{K}^{+}$deprivation and re-supplied 
with $20 \mathrm{mM} \mathrm{K}^{+}$for $3 \mathrm{~h}$ before sap sampling. The plant growth protocol ensured that, when compared with their wild type control, all the plants displayed the same visual phenotype and no statistically significant difference in biomass.

a, $\mathrm{K}^{+}$content in the collected phloem sap samples ( $\mu$ mole per plant).

b, $\mathrm{K}^{+}$concentration in the collected xylem sap samples.

Statistical analysis: Means $\pm \mathrm{SE} ; \mathrm{n}=3$. Student's t tests. ${ }^{*}, \mathrm{P}<0.05 ; * \star, P<0.01$

\section{Fig. 6}
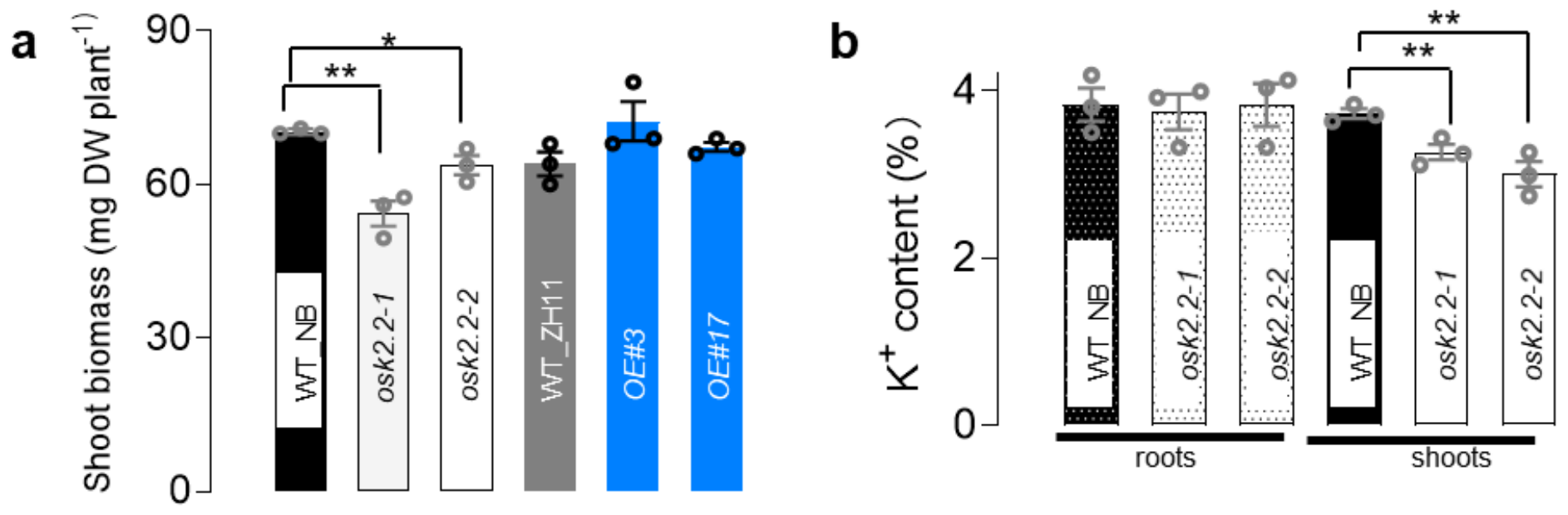

C
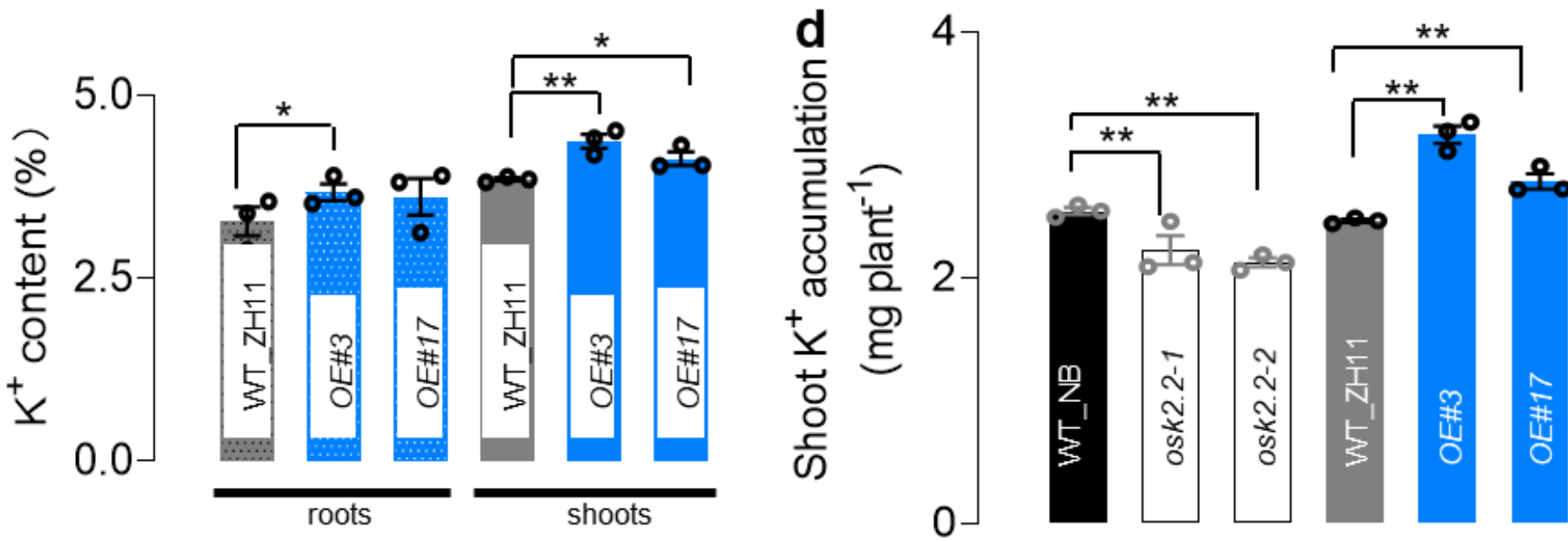

Figure 6

Contribution of OsK2.2 to shoot biomass production and $\mathrm{K}^{+}$accumulation.

Ten-day-old seedlings (Nipponbare wild type and osk2.2-1 and osk2.2-2 KO mutants, and Zhonghua-11 wild type and overexpressors $O E \# 3$ and $O E \# 17$, see legend to Fig. 5) were hydroponically grown for 7 
further days in $10 \mathrm{mM} \mathrm{K}^{+}$and then sampled for biomass and $\mathrm{K}^{+}$content measurements.

a, Shoot biomass. b, Root and shoot $\mathrm{K}^{+}$contents in the Nipponbare plants.

c, Root and shoot $\mathrm{K}^{+}$contents in the Zhonghua-11 plants. $\mathbf{d}$, Total $\mathrm{K}^{+}$accumulation in shoots (computed from shoot biomass and $\mathrm{K}^{+}$contents) in the Nipponbare and Zhonghua-11 plants.

Means $\pm S E(n=3)$. Statistical significance: Student's $t$ tests. *, $P<0.05 ; * \star, P<0.01$.

\section{Fig. 7}
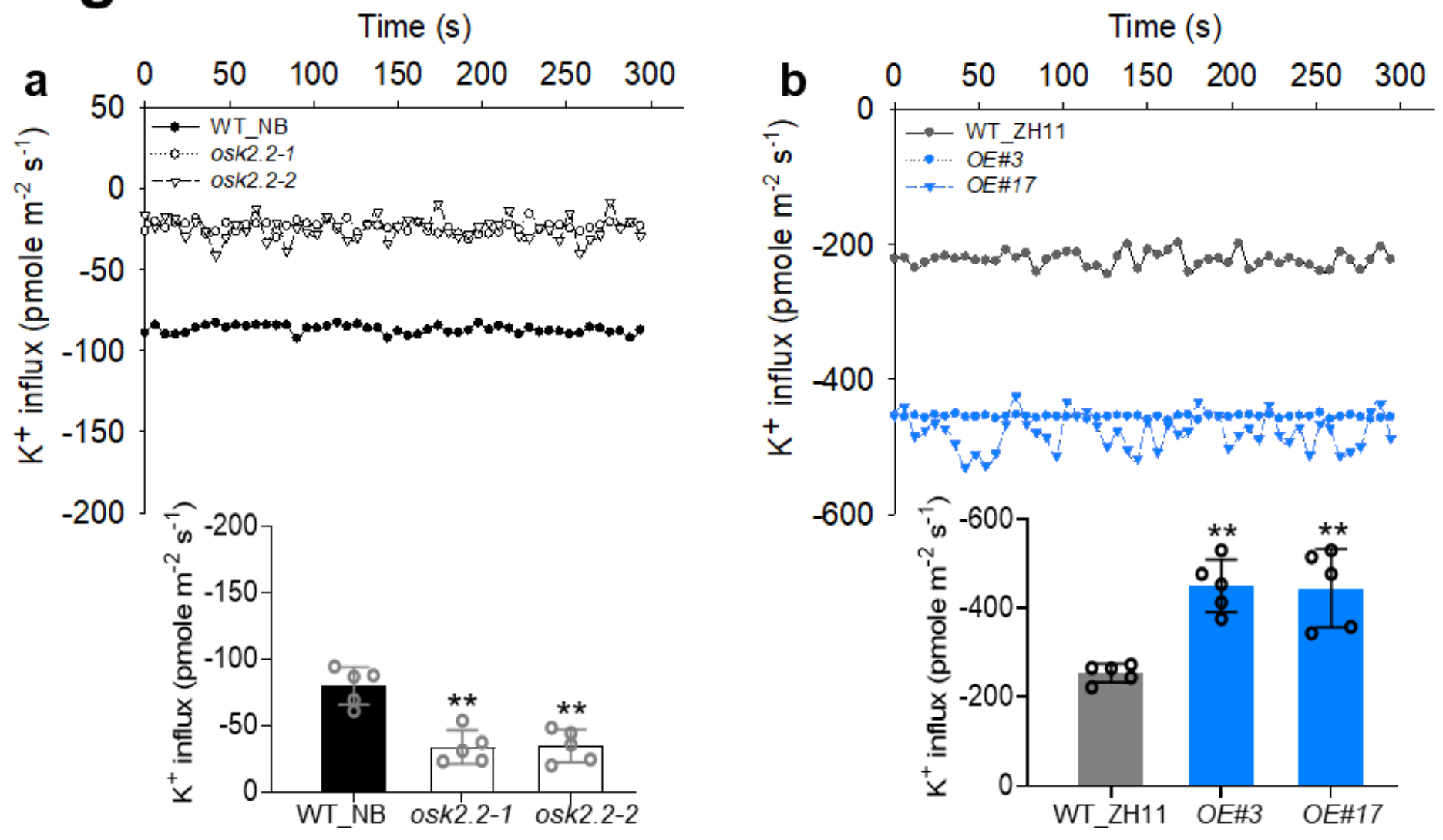

Figure 7

OsK2.2 functional expression facilitates net $\mathrm{K}^{+}$uptake by root periphery cells.

Net $\mathrm{K}^{+}$influx was measured in rice roots from 10-day-old plants using $\mathrm{K}^{+}$-selective electrodes ("noninvasive micro-test (NMT) technique") at ca. $2 \mathrm{~mm}$ from the root apex (region identified as displaying the largest rate of net $\mathrm{K}^{+}$influx) during a time course of $5 \mathrm{~min}$ in a bath solution containing $1 \mathrm{mM} \mathrm{K}^{+}$.

a, Nipponbare KO mutant plants (osk2.2-1 and osk2.2-2 lines) and the corresponding wildtype plants (WT_NB).

b, Zhonghua-11 OsK2.2 overexpressors (OE\#3 and OE\#17lines) and the corresponding wildtype plants. 
Insets: means $\pm \mathrm{SE} ; \mathrm{n}=5$. Statistical significance: Student's t tests. ${ }^{*}, \mathrm{P}<0.05 ;{ }^{*}, \mathrm{P}<0.01$.

Fig. 8

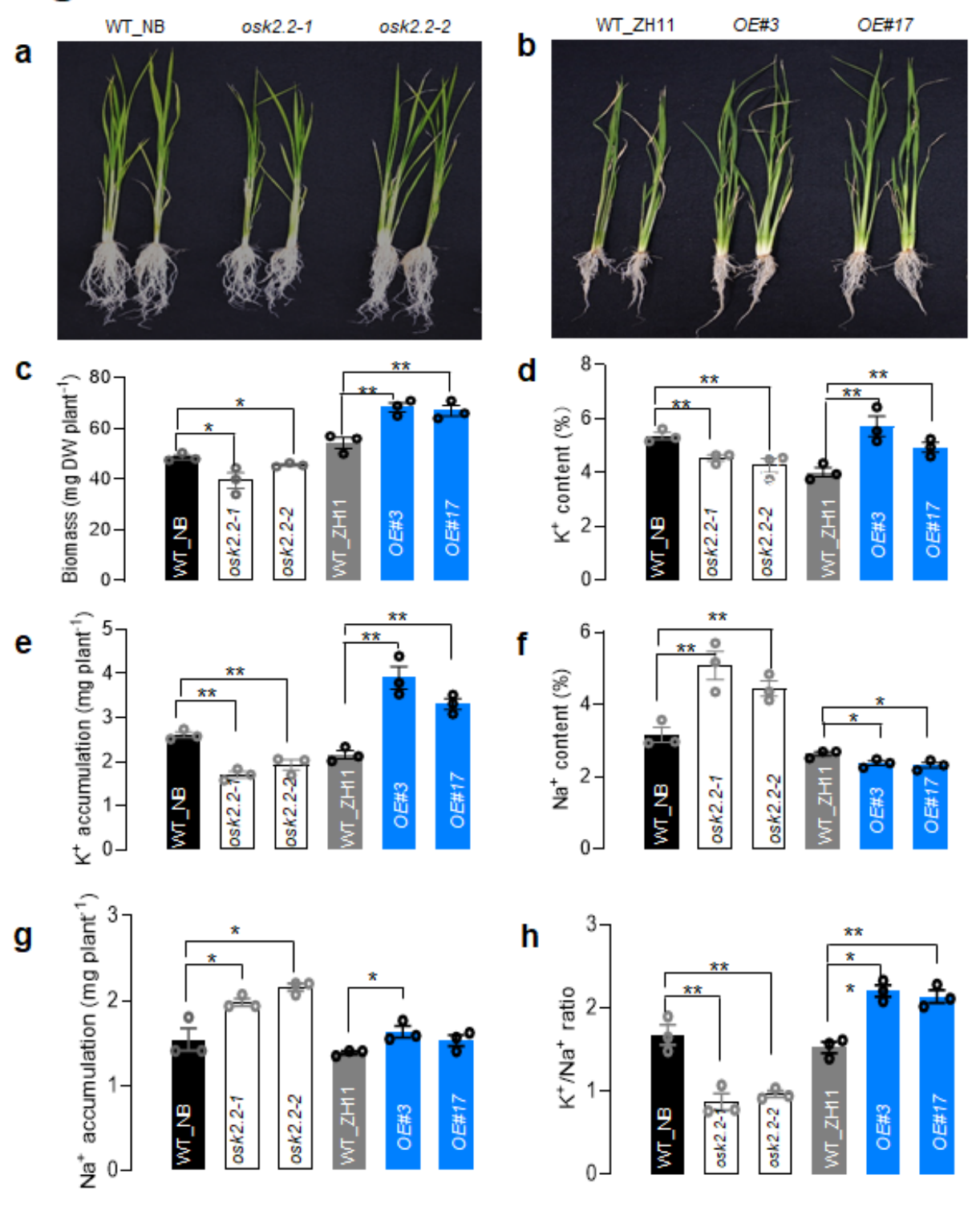

Figure 8

OsK2.2 contributes to rice adaptation to saline conditions.

Ten-day old rice seedlings grown in IRRI nutrient solution $\left(1 \mathrm{mM} \mathrm{K}^{+}\right)$were grown for 14 further days in the presence of $100 \mathrm{mM} \mathrm{NaCl}$.

a,b, Photographs of $\mathrm{NaCl}$ stressed plants. Nipponbare KO mutant plants (osk2.2-1 and osk2.2-2 lines) and corresponding control wildtype plants (WT_NB) are shown in (a), and Zhonghua-11 OsK2.2 overexpressors (OE\#3 and OE\#17lines) and corresponding control wildtype plants (WT_ZH11) in (b). 
c, Shoot biomass at the end of the $100 \mathrm{mM} \mathrm{NaCl}$ treatment. d, Shoot $\mathrm{K}^{+}$contents at the end of the $\mathrm{NaCl}$ treatment. $\mathbf{e}$, Total accumulation of $\mathrm{K}^{+}$in shoots (computed from shoot biomass and shoot $\mathrm{K}^{+}$content). $\mathrm{f}$, Shoot $\mathrm{Na}^{+}$contents. $\mathbf{g}$, Total accumulation of $\mathrm{Na}^{+}$in shoots. $\mathbf{h}$, Deduced ratios of shoot $\mathrm{K}^{+}$content to shoot $\mathrm{Na}^{+}$content.

Means \pm SE $(n=3)$. Statistical analysis: Student's $t$ tests. ${ }^{*}, P<0.05 ; * \star, P<0.01$ 
Fig. 9
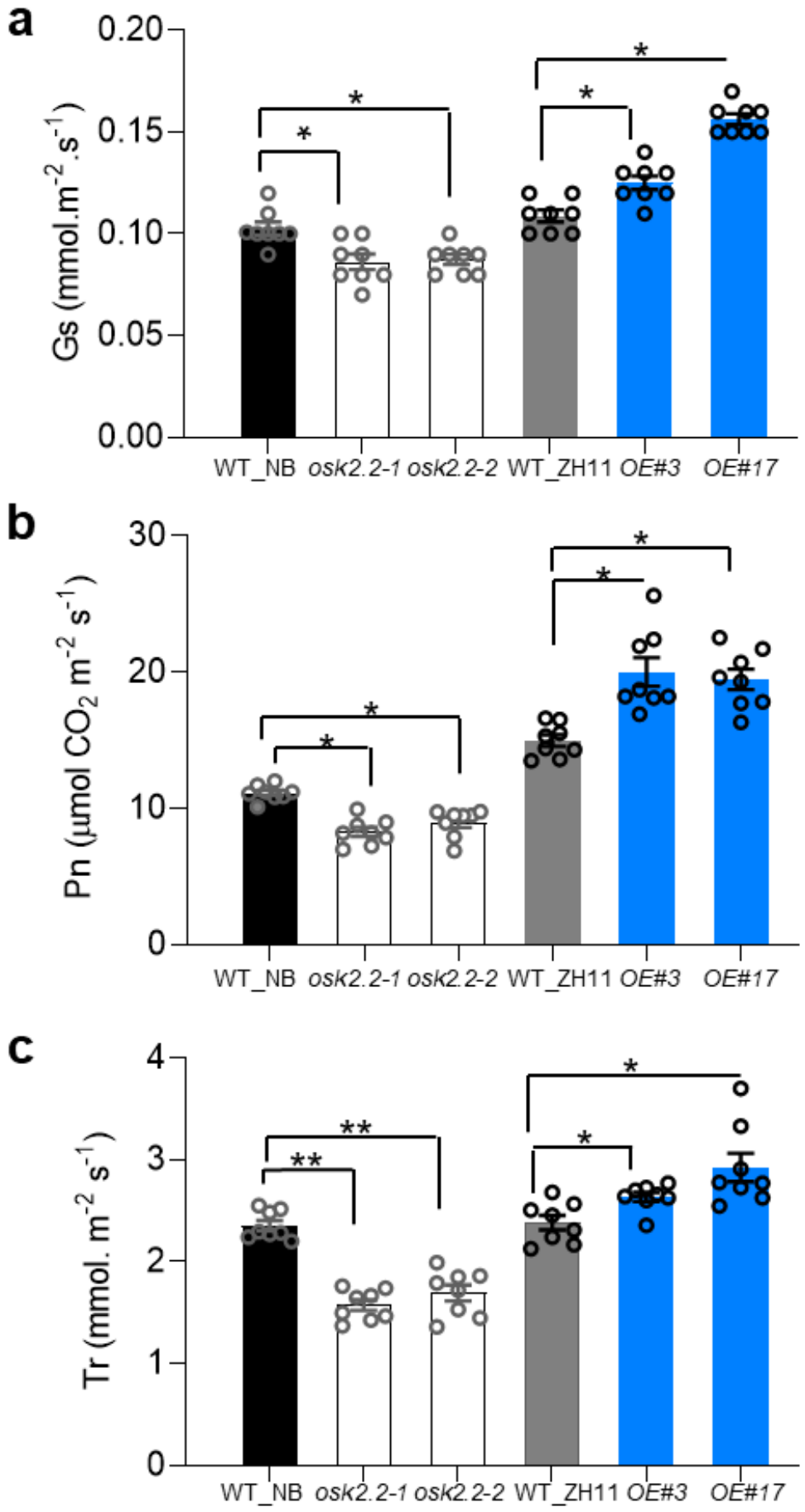

Figure 9

Stomatal conductance, net photosynthesis and transpiration are sensitive to the level of OsK2.2 expression.

Fourteen-day-old seedlings (Nipponbare wildtype and osk2.2-1 and osk2.2-2 KO mutants, and Zhonghua11 wildtype and overexpressors $O E \# 3$ and $O E \# 17$, see legend to Fig. 5) were subjected to $\mathrm{K}^{+}$depletion for 
3 days prior being resupplied with $20 \mathrm{mM} \mathrm{K}^{+}$(same experimental conditions as in Fig. 5 experiment). Gas exchange parameters were measured $2 \mathrm{~h}$ later using a LI-COR LI-6400 portable photosynthesis system. a, Stomatal conductance. $\mathbf{b}$, Net photosynthesis rate. $\mathbf{c}$, Transpiration rate. Data are means \pm SE $(n=8)$. Statistical analysis: Student's t tests. ${ }^{*}, \mathrm{P}<0.05 ; * \star, \mathrm{P}<0.01$.

\section{Fig. 10}

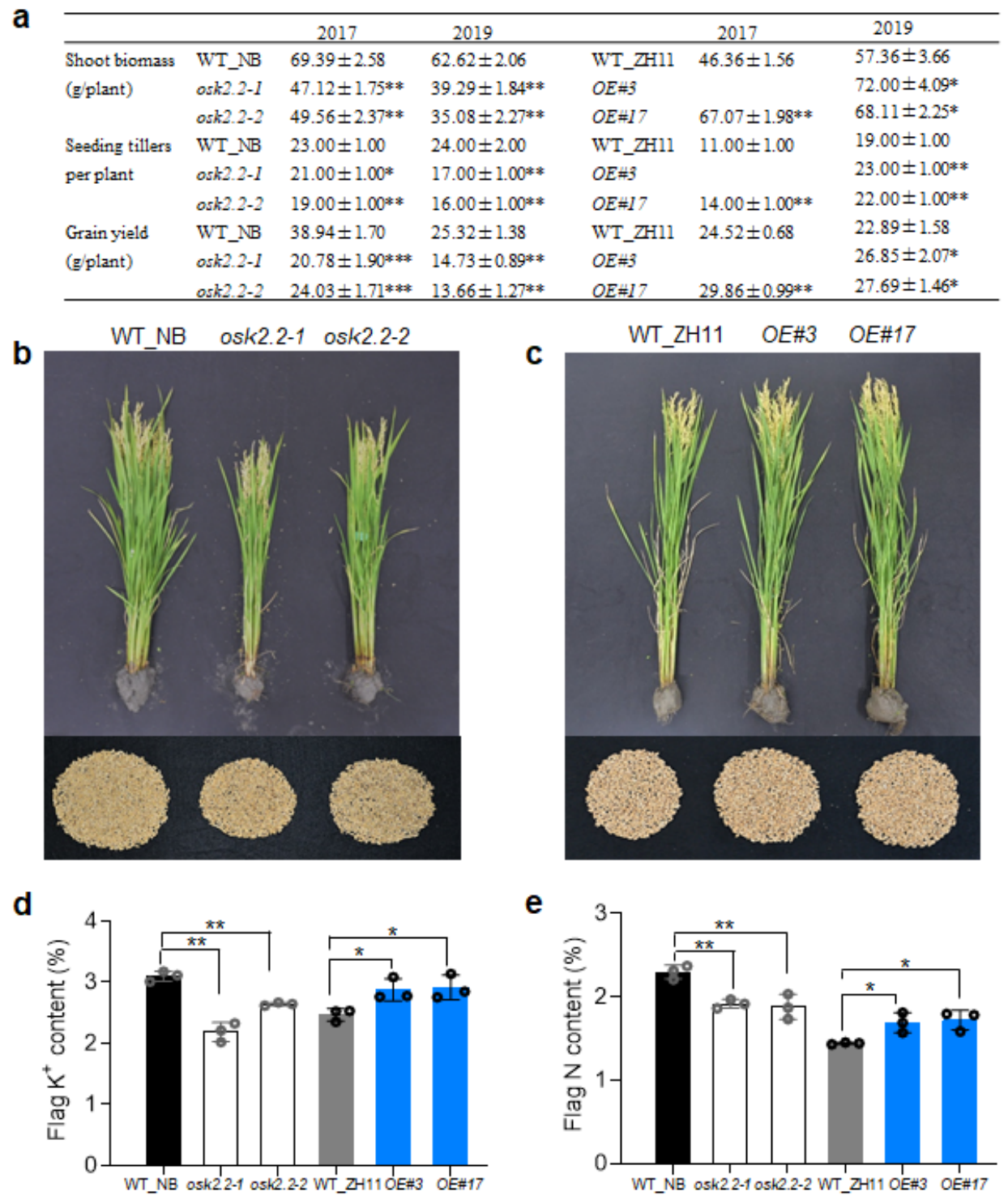

Figure 10

Functional expression of $O s K 2.2$ can significantly contribute to rice development and grain yield in field conditions.

Plants (Nipponbare wild type and osk2.2-1 and osk2.2-2 KO mutants, and Zhonghua-11 wild type and overexpressors $O E \# 3$ and $O E \# 17)$ were grown in field conditions with $\mathrm{K}, \mathrm{N}$ and $\mathrm{P}$ fertilization $\left(240 \mathrm{~kg} \mathrm{~K}{ }_{2} \mathrm{O}\right.$, 
$200 \mathrm{~kg} \mathrm{~N}$ and $90 \mathrm{~kg} \mathrm{P}_{2} \mathrm{O}_{5}$ per $\mathrm{hm}^{2}$ ).

a, Yield-related parameters (shoot biomass, number of seeding tillers per plant and grain yield per plant) were measured during two independent seasons (T3 and T4 generations for both the homozygous KO mutant and overexpressing lines). Means $\pm S E(n=15)$. Statistical analysis: Student's $t$ tests. *, $P<0.05$; $\star \star, P<0.01$.

b,c, Plant growth at the grain-filling stage and grain yield: representative photographs for Nipponbare (b) and Zhonghua-11 (c) plants.

d,e, $\mathrm{K}^{+}(\mathbf{d})$ and nitrogen $(\mathbf{e})$ contents of flag leaves determined at late grain-filling stage $(n=3, T 4$ generations). Data are means \pm SE. Statistical analysis: Student's $t$ tests; ${ }^{*}, P<0.05 ; * \star, P<0.01$.

\section{Supplementary Files}

This is a list of supplementary files associated with this preprint. Click to download.

- Supplementaryinformationfiles.docx 